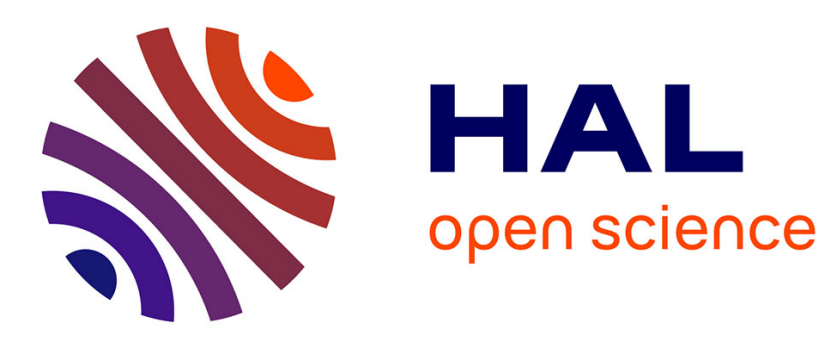

\title{
Ferrocenyl flavonoid-induced morphological modifications of endothelial cells and cytotoxicity against B16 murine melanoma cells
}

Jean-Philippe Monserrat, Keshri Nath Tiwari, Lionel Quentin, Pascal Pigeon, Gérard Jaouen, Anne Vessières, Guy G. Chabot, Elisabeth A. Hillard

\section{To cite this version:}

Jean-Philippe Monserrat, Keshri Nath Tiwari, Lionel Quentin, Pascal Pigeon, Gérard Jaouen, et al.. Ferrocenyl flavonoid-induced morphological modifications of endothelial cells and cytotoxicity against B16 murine melanoma cells. Journal of Organometallic Chemistry, 2013, 734 (SI), pp.78-85. 10.1016/j.jorganchem.2012.12.031 . hal-01230380

\section{HAL Id: hal-01230380 \\ https://hal.science/hal-01230380}

Submitted on 29 Mar 2021

HAL is a multi-disciplinary open access archive for the deposit and dissemination of scientific research documents, whether they are published or not. The documents may come from teaching and research institutions in France or abroad, or from public or private research centers.
L'archive ouverte pluridisciplinaire HAL, est destinée au dépôt et à la diffusion de documents scientifiques de niveau recherche, publiés ou non, émanant des établissements d'enseignement et de recherche français ou étrangers, des laboratoires publics ou privés. 


\title{
Ferrocenyl flavonoid-induced morphological modifications of endothelial cells and cytotoxicity against B16 murine melanoma cells
}

Jean-Philippe Monserrat ${ }^{\mathrm{a}, \mathrm{b}, 1}$, Keshri Nath Tiwari ${ }^{\mathrm{a}, \mathrm{b}, 1}$, Lionel Quentin ${ }^{\mathrm{c}, \mathrm{d}, \mathrm{e}, \mathrm{f}}$, Pascal Pigeon ${ }^{\mathrm{a}, \mathrm{b}}$, Gérard Jaouen ${ }^{\mathrm{a}, \mathrm{b}}$, Anne Vessières $^{\mathrm{a}, \mathrm{b}}$, Guy G. Chabot ${ }^{\mathrm{c}, \mathrm{d}, \mathrm{e}, \mathrm{f}}$, Elizabeth A. Hillard ${ }^{\mathrm{a}, \mathrm{b}, *}$

${ }^{a}$ ENSCP Chimie ParisTech, Laboratoire Charles Friedel (LCF), 75005 Paris, France

${ }^{\mathrm{b}}$ CNRS, UMR 7223, 75005 Paris, France

${ }^{c}$ ENSCP Chimie ParisTech, Unité de Pharmacologie Chimique et Génétique et Imagerie (UPCGI), 75005 Paris, France

${ }^{\mathrm{d}}$ Université Paris Descartes, Faculty of Pharmacy, 75006 Paris, France

${ }^{\mathrm{e}}$ CNRS, UMR 8151, 75006 Paris, France

${ }^{\mathrm{f}}$ INSERM, U 1022, 75006 Paris, France

* Corresponding author. Present address: CNRS, CRPP, UPR 8641, F-33600 Pessac, France. Tel.: +33 (0)5 568456

24; fax: +33 (0)5 568456 00. E-mail address: hillard@crpp-bordeaux.cnrs.fr (E.A. Hillard).

${ }^{1}$ These authors contributed equally to the work.

\begin{abstract}
With the aim of improving the cytotoxic and vascular disrupting activities of flavonoids, several classes of ferrocenyl-modified flavonoids were prepared and tested on cancer and endothelial cells. Three ten-member series of ferrocenyl flavonoids: chalcones $((E)-1-(R-2$ '-hydroxyphenyl)-3-ferrocenylprop-2-en-1-ones), aurones $((Z)-R$-2-(ferrocenylidene) benzofuran-3-ones) and flavones ( $R$-2-ferrocenyl-chromen-4-ones) were synthesized by recently reported methods. Three ferrocenyl flavonols ( $R$-3-hydroxy-2-ferrocenyl-chromen4-ones) and four ferrocenyl flavanones (3-ferrocenylmethylidenyl- $R$-2-phenyl-chroman-4-ones) were also obtained. All compounds were evaluated for their cytotoxic effects on a cancer cell line (B16 murine melanoma) and for their morphological effects on endothelial cells (EAhy 926). Some interesting structureactivity relationships were disclosed: of all the compounds, the halogen-substituted aurones showed the best cytotoxic activity, with $\mathrm{IC}_{50}$ values ranging between 12 and $18 \mu \mathrm{M}$. Ferrocenyl flavonols and ferrocenyl flavanones with substitution in the 3-position ( $-\mathrm{OH}$ and $=\mathrm{C}-\mathrm{Fc}$ respectively) were not active against cancer or endothelial cells. Some of the ferrocenyl flavones caused the endothelial cells to adopt a round shape ("rounding up") at submicromolar concentrations, which can be predictive of vascular disrupting activity. The most morphologically active flavones showed only moderate cytotoxicity against cancer cells, indicating that they may primarily act as antivascular agents.
\end{abstract}

Keywords: Flavones; Aurones; Ferrocene; Cytotoxicity; Antivascular agents; Bioorganometallic chemistry

\section{Introduction}

Flavonoids are small molecules, widespread in the plant kingdom, consisting of two phenyl rings linked by a 3-carbon atom bridge, and represent the most common polyphenols in the human diet. They are currently enjoying enormous interest as nutraceuticals and drug candidates for diabetes, cognitive function, blood pressure, cancer, obesity, and other diseases [1]. Appearing with the first plants on earth and thus having accompanied the evolution of animal life, flavonoids interact with many biological pathways. In 
cancer research, flavonoids have been shown to inhibit various pathways related to drug resistance [2], apoptosis [3], division [4] and metastasis [5]. Currently, there is great interest in flavonoids as angiogenesis inhibitors. Flavonoids are an important class of vascular disrupting agents, an experimental treatment involving rapid destruction of the tumor vasculature and thus preventing oxygen and nutrient flow to the tumor [6]. One flavonoid, ASA404 (vadimezan), is currently undergoing Phase II clinical trials as a vascular disrupting agent [7]. ASA404 acts by disrupting the actin cytoskeleton of tumor vascular endothelial cells, making the vessels more permeable. When used in combination, this molecule has been shown to enhance the efficacy of carboplatin and paclitaxel [8].

Organometallic medicinal chemistry is one of the most mature and active areas in bioorganometallic chemistry [9], and related applications in enzyme inhibition, bioimaging, immunoassay, small molecule delivery, and in cellulo catalysis are growing topics [10]. The use of organometallic compounds in anticancer drug discovery continues to receive a great deal of attention [11], as such molecules are often more stable to hydrolysis than coordination compounds. The introduction of organometallics can alter the physicochemical characteristics of a bioactive compound by acting as a structural element [12], controlling hydrolysis kinetics, or modifying its lipophilicity [13]. Organometallic units can also add supplementary reactivity to the biomolecule by imparting redox properties [14] or acting as an alkylating function to proteins [15] or DNA [16]. From another point of view, bioligands can be used to vectorize cytotoxic metal moieties, bringing them into the mitochondria or nucleus, and allowing them to interact with receptors [17]. Ferrocene in particular has gained considerable interest as a substituent in medicinal chemistry due to its stability in aqueous and aerobic media, its lipophilicity, its ease of derivation, and its redox activity [18]. Some ferrocene derivatives have shown promising activity against proteins, cells or organisms associated with diseases including fungal and bacterial infections [19], malaria [20], HIV [21], and cancer [22].

While there are several examples of biologically active flavonoid metal complexes [23], few examples of organometallic flavonoids, apart from ferrocenyl chalcones [24], can be found in the literature [25]. Notably, however, ruthenium arenes coordinated to flavonols recently displayed particularly interesting cytotoxic activity against $\mathrm{CH} 1$ ovarian cells, possibly due to binding to DNA accompanied by topoisomerase IIa inhibitory activity [26]. As part of our ongoing project studying ferrocene-modified biomolecules, we have recently obtained access to a variety of ferrocenyl flavonoids [27]. We now present our studies on the antiproliferative effects on B16 murine melanoma and the potential vascular disrupting effects on the EAhy 926 endothelial cell model of a number of ferrocenyl chalcones, aurones, flavones, flavanols and flavanones. Some interesting structure-activity relationships were observed and a clear differential activity between the cytotoxic effects on cancer cells and the morphological effects on endothelial cells was observed for certain compounds, suggesting that these activities are not correlated.

\section{Material and methods}

\subsection{General remarks}

All reactions were carried out under argon. THF was distilled over sodium/benzophenone. All other chemical reagents and solvents were used without further purification. Silica gel chromatography was performed with Merck $60(40-63 \mu \mathrm{m})$ silica. ${ }^{1} \mathrm{H}$ and ${ }^{13} \mathrm{C}$ NMR spectra were recorded with a $300 \mathrm{or} 400 \mathrm{MHz}$ Bruker Avance spectrometer, and $\delta$ are given in ppm and referenced to the residual solvent peaks $\left({ }^{1} \mathrm{H}, \delta 7.26\right.$ and ${ }^{13} \mathrm{C}\left\{{ }^{1} \mathrm{H}\right\}, \delta 77.1$ for $\mathrm{CDCl}_{3}$ and ${ }^{1} \mathrm{H}, \delta 2.05$ and ${ }^{13} \mathrm{C}\left\{{ }^{1} \mathrm{H}\right\}, \delta 29.7$ for acetone $\mathrm{d}_{6}$ ). Mass spectra were measured on a Thermoscientific ITQ1100 spectrometer using the direct exposure probe method by the mass spectrometry service at ENSCP Chimie ParisTech. High resolution mass spectra were obtained by ESI/ESCI-TOF using a Waters LCT Premier XE or a Thermo Fischer LTQOrbitrap XL at the Institut Parisien de Chimie Moléculaire (IPCM). Melting points were determined using an Electrothermal 9100 apparatus. IR data were collected on a JASCO FT/IR-4100 using a KBr pellet. The purity of all compounds (>98\%) was confirmed using a Shimadzu HPLC LC20 series equipped with LC-20AB pump and SPD-20A UV detector prior to biological testing. 


\subsection{Synthesis}

The synthesis and characterization of the ferrocenyl chalcones $\mathbf{1 a - j}$ and ferrocenyl aurones $\mathbf{2 a - j}$ have been previously reported [28].

\subsubsection{General synthesis of ferrocenyl flavones $\mathbf{3 a - j}$}

Ferrocenyl aurone $(30 \mathrm{mg})$ and potassium cyanide (1.5 equiv) were dissolved in ethanol $(25 \mathrm{~mL})$ and stirred at reflux for $2 \mathrm{~h}$. The solution was cooled to rt and poured into an aqueous solution of $1 \mathrm{M} \mathrm{NaOH}$ $(100 \mathrm{~mL})$. The mixture was extracted with EtOAc $(3 \times 50 \mathrm{~mL})$ and washed with water. The organic phase was dried over $\mathrm{MgSO}_{4}$, filtered, and evaporated. The crude product was purified using a silica gel column, with 60/40 petroleum ether/ethyl acetate as an eluent and was isolated as an orange solid. Characterization of $\mathbf{3 a}, \mathbf{3 c}$ and $\mathbf{3 h}$ has been previously reported [27].

2.2.1.1. 6-chloro-2-ferrocenyl-chromen-4-one, 3b. Yield: 72\%; $\mathrm{mp} 202{ }^{\circ} \mathrm{C}, v_{\max } / \mathrm{cm}^{-1} 1639(\mathrm{C}=\mathrm{O}), 1608$ $(\mathrm{C}=\mathrm{C}), \delta_{\mathrm{H}}\left(300 \mathrm{MHz} ; \mathrm{CDCl}_{3}\right) 4.17\left(\mathrm{~s}, 5 \mathrm{H}, \mathrm{C}_{5} \mathrm{H}_{5}\right), 4.54\left(\mathrm{~s}, 2 \mathrm{H}, \mathrm{C}_{5} \mathrm{H}_{4}\right), 4.85\left(\mathrm{~s}, 2 \mathrm{H}, \mathrm{C}_{5} \mathrm{H}_{4}\right), 6.45(\mathrm{~s}, 1 \mathrm{H}$, vinyl), $7.45\left(\mathrm{~d}, 1 \mathrm{H}, J=8.5 \mathrm{~Hz}, \mathrm{C}_{6} \mathrm{H}_{3}\right), 7.59\left(\mathrm{~d}, 1 \mathrm{H}, \mathrm{J}=8.5 \mathrm{~Hz}, \mathrm{C}_{6} \mathrm{H}_{3}\right), 8.16\left(\mathrm{~s}, 1 \mathrm{H}, \mathrm{C}_{6} \mathrm{H}_{3}\right) . \delta_{\mathrm{C}}\left(75 \mathrm{MHz} ; \mathrm{CDCl}_{3}\right) 67.5$ $\left(\mathrm{C}_{5} \mathrm{H}_{4}\right), 70.3\left(\mathrm{C}_{5} \mathrm{H}_{5}\right), 71.6\left(\mathrm{C}_{5} \mathrm{H}_{4}\right), 74.5\left(\mathrm{C}_{5} \mathrm{H}_{4}\right.$ ipso $), 105.6(\mathrm{C}=\mathrm{C}), 111.6\left(\mathrm{C}_{6} \mathrm{H}_{3}\right), 119.4\left(\mathrm{C}_{6} \mathrm{H}_{3}\right), 125.1\left(\mathrm{C}_{6} \mathrm{H}_{3}\right)$, $130.9\left(\mathrm{C}_{6} \mathrm{H}_{3}\right), 133.4\left(\mathrm{C}_{6} \mathrm{H}_{3}\right), 154.5\left(\mathrm{C}_{6} \mathrm{H}_{3}\right), 168.7(\mathrm{C}=\mathrm{C}), 176.2(\mathrm{C}=\mathrm{O})$. HRMS (ESI) calcd. for $\mathrm{C}_{19} \mathrm{H}_{14} \mathrm{ClFeO}_{2}{ }^{+}$: 365.0032, found: 365.0027 .

2.2.1.2. 6,8-difluoro-2-ferrocenyl-chromen-4-one, 3d. Yield: $60 \% ; \mathrm{mp} 155{ }^{\circ} \mathrm{C}, v_{\max } / \mathrm{cm}^{-1} 1639(\mathrm{C}=\mathrm{O}), 1585$ $(\mathrm{C}=\mathrm{C}), \delta_{\mathrm{H}}\left(400 \mathrm{MHz} ; \mathrm{CDCl}_{3}\right) 4.18\left(\mathrm{~s}, 5 \mathrm{H}, \mathrm{C}_{5} \mathrm{H}_{5}\right), 4.56\left(\mathrm{t}, 2 \mathrm{H}, J=1.9 \mathrm{~Hz}, \mathrm{C}_{5} \mathrm{H}_{4}\right), 4.88(\mathrm{t}, 2 \mathrm{H}, J=1.9 \mathrm{~Hz}$, $\left.\mathrm{C}_{5} \mathrm{H}_{4}\right), 6.44\left(\mathrm{~s}, 1 \mathrm{H}\right.$, vinyl), $7.18\left(\mathrm{dd}, 1 \mathrm{H}, J=8.8 \mathrm{~Hz}, J=2.4 \mathrm{~Hz}, \mathrm{C}_{6} \mathrm{H}_{2}\right), 7.63\left(\mathrm{~d}, 1 \mathrm{H}, J=8.8 \mathrm{~Hz}, \mathrm{C}_{6} \mathrm{H}_{2}\right) . \delta_{\mathrm{C}}$ $\left(100 \mathrm{MHz} ; \mathrm{CDCl}_{3}\right) 67.7\left(\mathrm{C}_{5} \mathrm{H}_{4}\right), 70.5\left(\mathrm{C}_{5} \mathrm{H}_{5}\right), 71.9\left(\mathrm{C}_{5} \mathrm{H}_{4}\right), 74.2\left(\mathrm{C}_{5} \mathrm{H}_{4}\right.$ ipso $), 105.4(\mathrm{C}=\mathrm{C}), 106.0\left(\mathrm{dd},{ }^{2} \mathrm{~J}=\right.$ $\left.23.7 \mathrm{~Hz},{ }^{4} J=4.0 \mathrm{~Hz}, \mathrm{C}_{6} \mathrm{H}_{2}\right) 108.7\left(\mathrm{dd},{ }^{3} J=20.1 \mathrm{~Hz},{ }^{3} J=28.5 \mathrm{~Hz}, \mathrm{C}_{6} \mathrm{H}_{2}\right), 126.8\left(\mathrm{~s}, \mathrm{C}_{6} \mathrm{H}_{2}\right), 141.9\left(\mathrm{~d},{ }^{2} J=9.4\right.$ $\left.\mathrm{Hz}, \mathrm{C}_{6} \mathrm{H}_{2}\right), 151.3\left(\mathrm{dd},{ }^{1} J=256 \mathrm{~Hz},{ }^{3} J=11.6 \mathrm{~Hz}, \mathrm{C}_{6} \mathrm{H}_{2}\right), 158.3\left(\mathrm{dd},{ }^{1} J=249 \mathrm{~Hz},{ }^{3} J=9.7 \mathrm{~Hz}, \mathrm{C}_{6} \mathrm{H}_{2}\right), 168.7$ $(\mathrm{C}=\mathrm{C}), 176.0(\mathrm{C}=\mathrm{O})$. HRMS (ESI) calcd. for $\mathrm{C}_{19} \mathrm{H}_{13} \mathrm{~F}_{2} \mathrm{FeO}_{2}{ }^{+}$: 367.0233, found: 367.0244.

2.2.1.3. 6,8-dichloro-2-ferrocenyl-chromen-4-one, 3e. Yield: $60 \% ; \mathrm{mp} 193{ }^{\circ} \mathrm{C}, v_{\max } / \mathrm{cm}^{-1} 1646(\mathrm{C}=\mathrm{O}), 1612$ $(\mathrm{C}=\mathrm{C}), \delta_{\mathrm{H}}\left(300 \mathrm{MHz} ; \mathrm{CDCl}_{3}\right) 4.18\left(\mathrm{~s}, 5 \mathrm{H}, \mathrm{C}_{5} \mathrm{H}_{5}\right), 4.58\left(\mathrm{t}, 2 \mathrm{H}, J=1.9 \mathrm{~Hz}, \mathrm{C}_{5} \mathrm{H}_{4}\right), 4.88(\mathrm{t}, 2 \mathrm{H}, J=1.9 \mathrm{~Hz}$, $\left.\mathrm{C}_{5} \mathrm{H}_{4}\right), 6.44\left(\mathrm{~s}, 1 \mathrm{H}\right.$, vinyl), $7.71\left(\mathrm{~d}, 1 \mathrm{H}, J=1.8 \mathrm{~Hz}, \mathrm{C}_{6} \mathrm{H}_{2}\right), 8.07\left(\mathrm{~d}, 1 \mathrm{H}, J=1.8, \mathrm{C}_{6} \mathrm{H}_{2}\right) . \delta_{\mathrm{C}}\left(75 \mathrm{MHz} ; \mathrm{CDCl}_{3}\right)$; $67.7\left(\mathrm{C}_{5} \mathrm{H}_{4}\right), 70.4\left(\mathrm{C}_{5} \mathrm{H}_{5}\right), 71.9\left(\mathrm{C}_{5} \mathrm{H}_{4}\right), 74.1\left(\mathrm{C}_{5} \mathrm{H}_{4}\right.$ ipso $), 105.3(\mathrm{C}=\mathrm{C}), 123.5\left(\mathrm{C}_{6} \mathrm{H}_{2}\right), 123.9\left(\mathrm{C}_{6} \mathrm{H}_{2}\right), 125.9$ $\left(\mathrm{C}_{6} \mathrm{H}_{2}\right), 130.5\left(\mathrm{C}_{6} \mathrm{H}_{2}\right), 133.3\left(\mathrm{C}_{6} \mathrm{H}_{2}\right), 150.4\left(\mathrm{C}_{6} \mathrm{H}_{2}\right), 168.9(\mathrm{C}=\mathrm{C}), 175.4(\mathrm{C}=\mathrm{O})$. HRMS (ESI) calcd. for $\mathrm{C}_{19} \mathrm{H}_{13} \mathrm{Cl}_{2} \mathrm{FeO}_{2}^{+}$: 398.9642, found: 398.9642 .

2.2.1.4. 6,8-dibromo-2-ferrocenyl-chromen-4-one, $3 f$. Yield: $55 \% ; \mathrm{mp} 194{ }^{\circ} \mathrm{C}, v_{\max } / \mathrm{cm}^{-1} 1643(\mathrm{C}=\mathrm{O}), 1612$ $(\mathrm{C}=\mathrm{C}), \delta_{\mathrm{H}}\left(300 \mathrm{MHz} ; \mathrm{CDCl}_{3}\right) 4.22\left(\mathrm{~s}, 5 \mathrm{H}, \mathrm{C}_{5} \mathrm{H}_{5}\right), 4.59\left(\mathrm{t}, 2 \mathrm{H}, J=1.9 \mathrm{~Hz}, \mathrm{C}_{5} \mathrm{H}_{4}\right), 4.93(\mathrm{t}, 2 \mathrm{H}, J=1.9 \mathrm{~Hz}$, $\mathrm{C}_{5} \mathrm{H}_{4}$ ), 6.44 (s, $1 \mathrm{H}$, vinyl), $8.01\left(\mathrm{~d}, 1 \mathrm{H}, J=2.4 \mathrm{~Hz}, \mathrm{C}_{6} \mathrm{H}_{2}\right), 8.27\left(\mathrm{~d}, 1 \mathrm{H}, J=2.4 \mathrm{~Hz}, \mathrm{C}_{6} \mathrm{H}_{2}\right) . \delta_{\mathrm{C}}(75 \mathrm{MHz}$; $\left.\mathrm{CDCl}_{3}\right), 67.8\left(\mathrm{C}_{5} \mathrm{H}_{4}\right), 70.4\left(\mathrm{C}_{5} \mathrm{H}_{5}\right), 71.8\left(\mathrm{C}_{5} \mathrm{H}_{4}\right), 74.1\left(\mathrm{C}_{5} \mathrm{H}_{4}\right.$ ipso $), 105.2(\mathrm{C}=\mathrm{C}), 112.5\left(\mathrm{C}_{6} \mathrm{H}_{2}\right), 118.1\left(\mathrm{C}_{6} \mathrm{H}_{2}\right)$, $127.8\left(\mathrm{C}_{6} \mathrm{H}_{2}\right), 136.6\left(\mathrm{C}_{6} \mathrm{H}_{2}\right), 138.8\left(\mathrm{C}_{6} \mathrm{H}_{2}\right), 151.7\left(\mathrm{C}_{6} \mathrm{H}_{2}\right), 169.0(\mathrm{C}=\mathrm{C}), 175.3(\mathrm{C}=\mathrm{O})$. HRMS (ESI) calcd. for $\mathrm{C}_{19} \mathrm{H}_{13}{ }^{79} \mathrm{Br}_{2} \mathrm{FeO}_{2}{ }^{+}$: 486.8632, found: 486.8629.

2.2.1.5. 7-methoxy-2-ferrocenyl-chromen-4-one, 3g. Yield: 70\%; $\mathrm{mp} 174{ }^{\circ} \mathrm{C}, v_{\max } / \mathrm{cm}^{-1} 1631(\mathrm{C}=\mathrm{O}), 1604$ $(\mathrm{C}=\mathrm{C}), \delta_{\mathrm{H}}\left(300 \mathrm{MHz} ; \mathrm{CDCl}_{3}\right) 3.93(\mathrm{~s}, 3 \mathrm{H}, \mathrm{OMe}) 4.17\left(\mathrm{~s}, 5 \mathrm{H}, \mathrm{C}_{5} \mathrm{H}_{5}\right), 4.50\left(\mathrm{t}, 2 \mathrm{H}, J=1.8 \mathrm{~Hz}, \mathrm{C}_{5} \mathrm{H}_{4}\right), 4.83(\mathrm{t}$, $\left.2 \mathrm{H}, J=1.8 \mathrm{~Hz}, \mathrm{C}_{5} \mathrm{H}_{4}\right), 6.40$ ( $\mathrm{s}, 1 \mathrm{H}$, vinyl), $6.89\left(\mathrm{~d}, 1 \mathrm{H}, J=2.3 \mathrm{~Hz}, \mathrm{C}_{6} \mathrm{H}_{3}\right), 6.95(\mathrm{dd}, 1 \mathrm{H}, J=2.3 \mathrm{~Hz}, J=8.9$ $\left.\mathrm{Hz}, \mathrm{C}_{6} \mathrm{H}_{3}\right), 8.10\left(\mathrm{~d}, 1 \mathrm{H}, J=8.9 \mathrm{~Hz}, \mathrm{C}_{6} \mathrm{H}_{3}\right) . \delta_{\mathrm{C}}\left(75 \mathrm{MHz} ; \mathrm{CDCl}_{3}\right) 55.6(\mathrm{OMe}), 67.1\left(\mathrm{C}_{5} \mathrm{H}_{4}\right), 70.0\left(\mathrm{C}_{5} \mathrm{H}_{5}\right), 71.0$ $\left(\mathrm{C}_{5} \mathrm{H}_{4}\right), 75.1\left(\mathrm{C}_{5} \mathrm{H}_{4}\right.$ ipso $), 100.1\left(\mathrm{C}_{6} \mathrm{H}_{3}\right), 105.4(\mathrm{C}=\mathrm{C}), 113.7\left(\mathrm{C}_{6} \mathrm{H}_{3}\right), 117.7\left(\mathrm{C}_{6} \mathrm{H}_{3}\right), 126.7\left(\mathrm{C}_{6} \mathrm{H}_{3}\right), 157.7$ $\left(\mathrm{C}_{6} \mathrm{H}_{3}\right), 163.6\left(\mathrm{C}_{6} \mathrm{H}_{3}\right), 167.4(\mathrm{C}=\mathrm{C}), 176.8(\mathrm{C}=\mathrm{O})$. HRMS (ESI) calcd. for $\mathrm{C}_{20} \mathrm{H}_{17} \mathrm{FeO}_{3}{ }^{+}$: 361.0520, found: 361.0527 . 
2.2.1.6. 5-methoxy-2-ferrocenyl-chromen-4-one, 3i. Yield: $68 \%$; $\mathrm{mp} 165{ }^{\circ} \mathrm{C}, v_{\max } / \mathrm{cm}^{-1} 1635(\mathrm{C}=\mathrm{O}), 1608$ $(\mathrm{C}=\mathrm{C}), \delta_{\mathrm{H}}(300 \mathrm{MHz}) 3.99(\mathrm{~s}, 3 \mathrm{H}, \mathrm{OMe}) 4.16\left(\mathrm{~s}, 5 \mathrm{H}, \mathrm{C}_{5} \mathrm{H}_{5}\right), 4.49\left(\mathrm{t}, 2 \mathrm{H}, J=1.8 \mathrm{~Hz}, \mathrm{C}_{5} \mathrm{H}_{4}\right), 4.82(\mathrm{t}, 2 \mathrm{H}, J=$ $\left.1.8 \mathrm{~Hz}, \mathrm{C}_{5} \mathrm{H}_{4}\right), 6.38\left(\mathrm{~s}, 1 \mathrm{H}\right.$, vinyl), $6.80\left(\mathrm{~d}, 1 \mathrm{H}, J=8.2 \mathrm{~Hz}, \mathrm{C}_{6} \mathrm{H}_{3}\right), 7.06\left(\mathrm{~d}, 1 \mathrm{H}, J=8.4 \mathrm{~Hz}, \mathrm{C}_{6} \mathrm{H}_{3}\right), 7.54(\mathrm{t}$, $\left.1 \mathrm{H}, J=8.4 \mathrm{~Hz}, \mathrm{C}_{6} \mathrm{H}_{3}\right) . \delta_{\mathrm{C}}\left(75 \mathrm{MHz} ; \mathrm{CDCl}_{3}\right) 55.3(\mathrm{OMe}), 67.0\left(\mathrm{C}_{5} \mathrm{H}_{4}\right), 69.9\left(\mathrm{C}_{5} \mathrm{H}_{5}\right), 70.9\left(\mathrm{C}_{5} \mathrm{H}_{4}\right), 74.8\left(\mathrm{C}_{5} \mathrm{H}_{4}\right.$ ipso), $106.1\left(\mathrm{C}_{6} \mathrm{H}_{3}\right), 107.7\left(\mathrm{C}_{6} \mathrm{H}_{3}\right), 109.7\left(\mathrm{C}_{6} \mathrm{H}_{3}\right), 114.4(\mathrm{C}=\mathrm{C}), 133.0\left(\mathrm{C}_{6} \mathrm{H}_{3}\right), 158.1\left(\mathrm{C}_{6} \mathrm{H}_{3}\right), 159.5\left(\mathrm{C}_{6} \mathrm{H}_{3}\right)$, $165.4(\mathrm{C}=\mathrm{C}), 177.4(\mathrm{C}=\mathrm{O})$. HRMS (ESI) calcd. for $\mathrm{C}_{20} \mathrm{H}_{17} \mathrm{FeO}_{3}{ }^{+}$: 361.0527, found: 361.0527.

2.2.1.7. 5,7-dimethoxy-2-ferrocenyl-chromen-4-one, 3j. Yield: $67 \% ; \mathrm{mp} 179{ }^{\circ} \mathrm{C}, v_{\max } / \mathrm{cm}^{-1} 1643(\mathrm{C}=\mathrm{O})$, $1608(\mathrm{C}=\mathrm{C}), \delta_{\mathrm{H}}\left(300 \mathrm{MHz} ; \mathrm{CDCl}_{3}\right) 3.90(\mathrm{~s}, 3 \mathrm{H}, \mathrm{OMe}), 3.93(\mathrm{~s}, 3 \mathrm{H}, \mathrm{OMe}), 4.14\left(\mathrm{~s}, 5 \mathrm{H}, \mathrm{C}_{5} \mathrm{H}_{5}\right), 4.45(\mathrm{t}, 2 \mathrm{H}, J$ $\left.=1.8 \mathrm{~Hz}, \mathrm{C}_{5} \mathrm{H}_{4}\right), 4.77\left(\mathrm{t}, 2 \mathrm{H}, J=1.8 \mathrm{~Hz}, \mathrm{C}_{5} \mathrm{H}_{4}\right), 6.30$ (s, $1 \mathrm{H}$, vinyl), $6.34\left(\mathrm{~d}, 1 \mathrm{H}, J=2.3 \mathrm{~Hz}, \mathrm{C}_{6} \mathrm{H}_{2}\right), 6.48(\mathrm{~d}$, $\left.1 \mathrm{H}, J=2.3 \mathrm{~Hz}, \mathrm{C}_{6} \mathrm{H}_{2}\right) . \delta_{\mathrm{C}}\left(75 \mathrm{MHz} ; \mathrm{CDCl}_{3}\right) 55.4(\mathrm{OMe}), 56.1(\mathrm{OMe}), 66.8\left(\mathrm{C}_{5} \mathrm{H}_{4}\right), 69.8\left(\mathrm{C}_{5} \mathrm{H}_{5}\right), 70.6\left(\mathrm{C}_{5} \mathrm{H}_{4}\right)$, 74.6 ( $\mathrm{C}_{5} \mathrm{H}_{4}$ ipso $), 92.4\left(\mathrm{C}_{6} \mathrm{H}_{2}\right), 95.6\left(\mathrm{C}_{6} \mathrm{H}_{2}\right), 106.9\left(\mathrm{C}_{6} \mathrm{H}_{2}\right), 109.0(\mathrm{C}=\mathrm{C}), 159.5\left(\mathrm{C}_{6} \mathrm{H}_{2}\right), 160.5\left(\mathrm{C}_{6} \mathrm{H}_{2}\right), 163.4$ $\left(\mathrm{C}_{6} \mathrm{H}_{2}\right), 164.5(\mathrm{C}=\mathrm{C}), 176.6(\mathrm{C}=\mathrm{O})$. HRMS (ESI) calcd. for $\mathrm{C}_{21} \mathrm{H}_{19} \mathrm{FeO}_{4}{ }^{+}$: 391.0622, found: 391.0633 .

\subsubsection{General synthesis of ferrocenyl flavonols $\mathbf{3 a}, \mathbf{3 b}$ and $\mathbf{3 h}$}

Carbonate buffer $(15 \mathrm{~mL})$ was added to a solution of ferrocenyl flavone $(40 \mathrm{mg})$ in $\mathrm{CH}_{2} \mathrm{Cl}_{2} /$ acetone $(2: 1,10 \mathrm{~mL})$ and stirred vigorously at $\mathrm{rt}$. A $5 \mathrm{~mL}$ aqueous solution of oxone $(0.6 \mathrm{~g})$ was added in four portions every $10 \mathrm{~min}$, and the $\mathrm{pH}$ of the solution was monitored to ensure that it remained alkaline. The consumption of starting material was followed by TLC. After the reaction was complete, the two phases were separated and the aqueous phase was extracted with $\mathrm{CH}_{2} \mathrm{Cl}_{2}(3 \times 50 \mathrm{~mL})$. The combined organic layer was washed with sodium thiosulfate solution, dried with $\mathrm{MgSO}_{4}$, filtered, and evaporated. The crude product was purified using a silica gel column with $80 / 20$ petroleum ether/ethyl acetate as eluent. Characterization of $\mathbf{3 a}$ and $\mathbf{3 b}$ has previously been reported [27].

2.2.2.1. 7-methoxy-3-hydroxy-2-ferrocenyl-chromen-4-one, 5h. Yield: 78\%; mp $208{ }^{\circ} \mathrm{C}, v_{\max } / \mathrm{cm}^{-1} 3255$ $(\mathrm{OH}), 1716(\mathrm{C}=\mathrm{O}), 1608(\mathrm{C}=\mathrm{C}), \delta_{\mathrm{H}}\left(400 \mathrm{MHz} ; \mathrm{CDCl}_{3}\right) 3.95(\mathrm{~s}, 3 \mathrm{H}, \mathrm{OMe}), 4.16\left(\mathrm{~s}, 5 \mathrm{H}, \mathrm{C}_{5} \mathrm{H}_{5}\right), 4.50\left(\mathrm{t},{ }^{3} \mathrm{~J}=\right.$ $\left.1.9 \mathrm{~Hz}, 2 \mathrm{H}, \mathrm{C}_{5} \mathrm{H}_{4}\right), 5.15\left(\mathrm{t},{ }^{3} J=1.9 \mathrm{~Hz}, 2 \mathrm{H}, \mathrm{C}_{5} \mathrm{H}_{4}\right), 6.70(\mathrm{~s}, 1 \mathrm{H}, \mathrm{OH}), 6.91\left(\mathrm{~d},{ }^{4} J=2.3 \mathrm{~Hz}, 1 \mathrm{H}, \mathrm{C}_{6} \mathrm{H}_{3}\right), 6.95$ $\left(\mathrm{dd},{ }^{3} J=8.9 \mathrm{~Hz}, 1 \mathrm{H}, \mathrm{C}_{6} \mathrm{H}_{3}\right), 8.08\left(\mathrm{~d},{ }^{3} J=8.9 \mathrm{~Hz}, 1 \mathrm{H}, \mathrm{C}_{6} \mathrm{H}_{3}\right) . \delta_{\mathrm{C}}\left(100 \mathrm{MHz} ; \mathrm{CDCl}_{3}\right) 55.9(\mathrm{OMe}), 68.4$ $\left(\mathrm{C}_{5} \mathrm{H}_{4}\right), 70.0\left(\mathrm{C}_{5} \mathrm{H}_{5}\right), 70.7\left(\mathrm{C}_{5} \mathrm{H}_{4}\right), 74.5\left(\mathrm{C}_{5} \mathrm{H}_{4}-\mathrm{C}\right.$ ipso $), 100.0\left(\mathrm{C}_{6} \mathrm{H}_{3}\right), 114.5\left(\mathrm{C}_{6} \mathrm{H}_{3}\right), 115.2\left(\mathrm{C}_{6} \mathrm{H}_{3}\right), 126.7$ $\left(\mathrm{C}_{6} \mathrm{H}_{3}\right), 136.5(\mathrm{C}=\mathrm{C}), 149.4(\mathrm{C}=\mathrm{C}), 157.3\left(\mathrm{C}_{6} \mathrm{H}_{3}\right), 163.8\left(\mathrm{C}_{6} \mathrm{H}_{3}\right), 171.2(\mathrm{C}=\mathrm{O})$. HRMS (ESI) calcd. for $\mathrm{C}_{20} \mathrm{H}_{16} \mathrm{FeO}_{4}^{+}$: 376.03980, found: 376.03939 .

\subsubsection{Synthesis of organic flavones $\mathbf{4 a - j}$}

Organic flavones $\mathbf{4 a - j}$ were synthesized by treating chalcones with $\mathrm{I}_{2}$ in DMSO according to a literature procedure [29]. The ${ }^{1} \mathrm{H}$ NMR spectra of 4-c [30], 4e [31], 4f [32], 4g, 4h [33], 4i [34] and 4j [35] were consistent with those found in the literature. Compound $\mathbf{4 d}$ was not found in the literature, and its structure was deduced from its ${ }^{1} \mathrm{H}$ NMR spectrum: $\delta_{\mathrm{H}}\left(300 \mathrm{MHz} ; \mathrm{CDCl}_{3}\right) 6.84(\mathrm{~s}, 1 \mathrm{H}$, vinyl), 7.23-7.30 (m, $\left.2 \mathrm{H}, \mathrm{C}_{6} \mathrm{H}_{5}\right)$, 7.54-7.56 (m, 1H, $\left.\mathrm{C}_{6} \mathrm{H}_{5}\right)$, 7.65-7.69 (m, 1H, $\left.\mathrm{C}_{6} \mathrm{H}_{2}\right)$, 7.93-7.96 (m, 2H, $\left.\mathrm{C}_{6} \mathrm{H}_{5} / \mathrm{C}_{6} \mathrm{H}_{2}\right)$.

\subsubsection{Synthesis of ferrocenyl flavanones}

Flavanone was purchased from Sigma Aldrich. Starting materials 7-methoxy-2-phenylchroman-4one, 5-methoxy-2-phenylchroman-4-one and 6-chloro-2-phenylchroman-4-one were prepared via treatment of the chalcone with pyridine in $\mathrm{MeOH}$ and water according to a reported procedure [36].

\subsubsection{3-ferrocenylmethylidenyl-2-phenylchroman-4-one, $\boldsymbol{6} \boldsymbol{a}$.}

2-phenylchroman-4-one (100 mg, $0.45 \mathrm{mmol})$ and ferrocene carboxaldehyde (96 mg, $0.45 \mathrm{mmol}$ ) were dissolved in $15 \mathrm{~mL} \mathrm{EtOH}$ in a round bottom flask. $\mathrm{HCl}$ gas, generated from concentrated sulfuric acid addition to $\mathrm{NaCl}$, was bubbled into the flavanone solution for $1 \mathrm{~h}$ and the solution was stirred at $0^{\circ}$ for $72 \mathrm{~h}$. The solution was extracted with $\mathrm{CH}_{2} \mathrm{Cl}_{2}$ and washed with a saturated solution of $\mathrm{NaCl}$ before being purified using a silica gel column using $\mathrm{CH}_{2} \mathrm{Cl}_{2}$ /petroleum ether (3:2) as an eluent. Yield $=18 \%$; mp $163{ }^{\circ} \mathrm{C}$, $v_{\max } / \mathrm{cm}^{-1} 1461,1596,1662, \delta_{\mathrm{H}}\left(300 \mathrm{MHz} ; \mathrm{CDCl}_{3}\right) 4.16\left(\mathrm{~s}, 5 \mathrm{H}, \mathrm{C}_{5} \mathrm{H}_{5}\right), 4.22\left(\mathrm{~s}, 1 \mathrm{H}, \mathrm{C}_{5} \mathrm{H}_{4}\right), 4.47(\mathrm{~s}, 2 \mathrm{H}$, 
$\left.\mathrm{C}_{5} \mathrm{H}_{4}\right), 4.55\left(\mathrm{~s}, 1 \mathrm{H}, \mathrm{C}_{5} \mathrm{H}_{4}\right), 6.62(\mathrm{~s}, 1 \mathrm{H}), 6.90(\mathrm{~m}, 2 \mathrm{H}), 7.27-7.37(\mathrm{~m}, 4 \mathrm{H}), 7.46(\mathrm{~m}, 2 \mathrm{H}), 7.94(\mathrm{~m}, 2 \mathrm{H}) . \delta_{\mathrm{C}}(75$ $\left.\mathrm{MHz} ; \mathrm{CDCl}_{3}\right)$ 68.5.2 $\left(\mathrm{C}_{5} \mathrm{H}_{5}\right), 69.5\left(\mathrm{C}_{5} \mathrm{H}_{4}\right), 71.8\left(\mathrm{C}_{5} \mathrm{H}_{4}\right), 72.0\left(\mathrm{C}_{5} \mathrm{H}_{4}, \mathrm{C}\right.$ ipso $)$ 74.0, 118.5, 121.6, 122.4, 127.6, $127.8,128.5,128.6,135.5,138.2,140.5,158.8,181.3$. MS (APEI) $m / z 420.18(\mathrm{M})^{+}$.

2.2.4.2. 3-ferrocenylmethylidenyl-7-methoxy-2-phenylchroman-4-one, $6 \boldsymbol{b}$. 7-methoxy-2-phenylchroman-4one (50 mg, $0.20 \mathrm{mmol})$ and ferrocene carboxaldehyde (43 mg, $0.20 \mathrm{mmol}$ ) were dissolved in $10 \mathrm{~mL} \mathrm{EtOH}$ in a round bottom flask and a spatula of $\mathrm{MgSO}_{4}$ was added. $\mathrm{HCl}$ gas was bubbled into the flavanone solution for $1 \mathrm{~h}$ and the solution was stirred at $\mathrm{rt}$ for $48 \mathrm{~h}$. The solution was extracted with $\mathrm{CH}_{2} \mathrm{Cl}_{2}$ and washed with a saturated solution of $\mathrm{NaCl}$ before being purified using a silica gel column with $50 / 50 \mathrm{CH}_{2} \mathrm{Cl}_{2} /$ petroleum ether as an eluent. Yield $=6 \% ; \delta_{\mathrm{H}}\left(300 \mathrm{MHz}\right.$; acetone $\left.\mathrm{d}_{6}\right): 4.05(\mathrm{~s}, 3 \mathrm{H}, \mathrm{OMe}), 4.40\left(\mathrm{~s}, 5 \mathrm{H}, \mathrm{C}_{5} \mathrm{H}_{5}\right), 4.50(\mathrm{~d}, J$ $\left.=1.6 \mathrm{~Hz}, 1 \mathrm{H}, \mathrm{C}_{5} \mathrm{H}_{4}\right), 4.75\left(\mathrm{t}, J=1.6 \mathrm{~Hz}, 2 \mathrm{H}, \mathrm{C}_{5} \mathrm{H}_{4}\right), 4.87\left(\mathrm{~d}, J=1.6 \mathrm{~Hz}, 1 \mathrm{H}, \mathrm{C}_{5} \mathrm{H}_{4}\right), 6.72(\mathrm{~d}, J=2.4 \mathrm{~Hz}, 1 \mathrm{H})$, $6.8(\mathrm{dd}, J=2.2 \mathrm{~Hz}, 8.8 \mathrm{~Hz}, 1 \mathrm{H}), 6.91(\mathrm{~s}, 1 \mathrm{H}), 7.58(\mathrm{~d}, J=7.3 \mathrm{~Hz}, 1 \mathrm{H}), 7.65(\mathrm{t}, J=1.6 \mathrm{~Hz}, 2 \mathrm{H}), 7.75(\mathrm{~d}, J=$ $7.3 \mathrm{~Hz}, 2 \mathrm{H}), 8.01(\mathrm{~d}, J=8.8 \mathrm{~Hz}, 1 \mathrm{H}), 8.1(\mathrm{~s}, 1 \mathrm{H})$. MS (APCI) $\mathrm{m} / z .451 .04(\mathrm{M}+\mathrm{H})^{+}$.

2.2.4.3. 3-ferrocenylmethylidenyl-6-methoxy-2-phenylchroman-4-one, 6c. 6-methoxy-2-phenylchroman-4one $(25 \mathrm{mg}, 0.1 \mathrm{mmol})$ and ferrocene carboxaldehyde $(22 \mathrm{mg}, 0.1 \mathrm{mmol})$ were dissolved in $15 \mathrm{~mL} \mathrm{EtOH}$ in a round bottom flask and a spatula of $\mathrm{MgSO}_{4}$ was added. $\mathrm{HCl}$ gas was bubbled into the flavanone solution for $5 \mathrm{~min}$ and the solution was stirred at $50{ }^{\circ} \mathrm{C}$ for $20 \mathrm{~h}$. The solution was extracted with $\mathrm{CH}_{2} \mathrm{Cl}_{2}$ and washed with a saturated solution of $\mathrm{NaCl}$ before being purified using a silica gel column with $50 / 50 \mathrm{CH}_{2} \mathrm{Cl}_{2}$ / petroleum ether as an eluent. Yield $=4 \% ; \delta_{\mathrm{H}}\left(300 \mathrm{MHz}\right.$; acetone $\left.\mathrm{d}_{6}\right) 4.06(\mathrm{~s}, 3 \mathrm{H}, \mathrm{OMe}), 4.16\left(\mathrm{~s}, 5 \mathrm{H}, \mathrm{C}_{5} \mathrm{H}_{5}\right)$, $4.41\left(\mathrm{t}, J=1.8 \mathrm{~Hz}, 1 \mathrm{H}, \mathrm{C}_{5} \mathrm{H}_{4}\right), 4.50\left(\mathrm{t}, J=1.8 \mathrm{~Hz}, 2 \mathrm{H}, \mathrm{C}_{5} \mathrm{H}_{4}\right), 4.67\left(\mathrm{t}, J=1.8 \mathrm{~Hz}, 1 \mathrm{H}, \mathrm{C}_{5} \mathrm{H}_{4}\right), 6.52(\mathrm{~s}, 1 \mathrm{H})$, $6.81(\mathrm{~s}, 1 \mathrm{H}), 7.16(\mathrm{~d}, J=2.8 \mathrm{~Hz}, 2 \mathrm{H}), 7.31(\mathrm{t}, J=1.2 \mathrm{~Hz}, 3 \mathrm{H}), 7.38(\mathrm{~d}, J=7.3 \mathrm{~Hz}, 2 \mathrm{H}), 7.77(\mathrm{~s}, 1 \mathrm{H}) . \mathrm{MS}$ (APCI) $\mathrm{m} / z 451.04(\mathrm{M}+\mathrm{H})^{+}$.

2.2.4.4. 3-ferrocenylmethylidenyl-6-chloro-2-phenylchroman-4-one, 6d. 6-chloro-2-phenylchroman-4-one $(150 \mathrm{mg}, 0.58 \mathrm{mmol})$ and ferrocenecarboxaldehyde $(124 \mathrm{mg}, 0.58 \mathrm{mmol})$ were dissolved in $20 \mathrm{~mL} \mathrm{EtOH}$ in a round bottom flask. $\mathrm{HCl}$ gas was bubbled into the flavanone solution for $1 \mathrm{~h}$ and the solution was stirred at $40^{\circ}$ for $72 \mathrm{~h}$. The solution was extracted with $\mathrm{CH}_{2} \mathrm{Cl}_{2}$ and washed with a saturated solution of $\mathrm{NaCl}$ before being purified using a silica gel column with 10/90 ethyl acetate/petroleum ether as an eluent. Yield $=15 \%$; $\operatorname{mp} 192{ }^{\circ} \mathrm{C}, v_{\max } / \mathrm{cm}^{-1} 1727,1662,1585,1461 ; \delta_{\mathrm{H}}\left(300 \mathrm{MHz} ; \mathrm{CDCl}_{3}\right) 4.13\left(\mathrm{t}, J=4.0 \mathrm{~Hz}, 5 \mathrm{H}, \mathrm{C}_{5} \mathrm{H}_{5}\right), 4.18(\mathrm{~d}$, $\left.J=1.5 \mathrm{~Hz}, 1 \mathrm{H}, \mathrm{C}_{5} \mathrm{H}_{4}\right), 4.47\left(\mathrm{t}, J=1.5 \mathrm{~Hz}, 2 \mathrm{H}, \mathrm{C}_{5} \mathrm{H}_{4}\right), 4.53\left(\mathrm{dd}, J=1.5 \mathrm{~Hz}, 3.8 \mathrm{~Hz}, 1 \mathrm{H}, \mathrm{C}_{5} \mathrm{H}_{4}\right), 6.60(\mathrm{~s}, 1 \mathrm{H})$, $6.87(\mathrm{~d}, J=8.8 \mathrm{~Hz}, 1 \mathrm{H}), 7.29-7.34(\mathrm{~m}, 3 \mathrm{H}), 7.36(\mathrm{~s}, 1 \mathrm{H}), 7.43-7.46(\mathrm{~m}, 2 \mathrm{H}), 7.88(\mathrm{~d}, J=2.6 \mathrm{~Hz}, 1 \mathrm{H}), 7.97$ (s, 1H). $\delta_{\mathrm{C}}\left(75 \mathrm{MHz} ; \mathrm{CDCl}_{3}\right)$ 68.4, 69.7, 72.1, 72.3, 74.2, 78.2, 120.2, 123.3, 126.9, 127.6, 128.7, 128.7, 135.3, 137.7, 141.5, 157.2, 180.2. MS (APCI) $m / z$. $454.94(\mathrm{M}+\mathrm{H})^{+}$.

\subsection{X-ray crystallography}

Intensity data were collected at $200 \mathrm{~K}$ with an Enraf-Nonius Kappa-CCD diffractometer equipped with a CCD two-dimensional detector. Data reduction was performed with DENZO/SCALEPACK. Data were corrected for Lorentz and polarization effects, and a semi-empirical absorption correction based on symmetry equivalent reflections was applied using SADABS. Lattice parameters were obtained from leastsquares analysis of 188 reflections. The structures were solved by direct methods and refined by full matrix least squares (5725 reflections/263 parameters), based on $\mathrm{F}^{2}$, using the Crystals software package. All nonhydrogen atoms were refined with anisotropic displacement parameters. All hydrogen atoms were located with geometrical restraints in riding mode.

\subsection{Biological assays}

\subsubsection{Cytotoxicity on cancer cells}

Murine B16 melanoma cells were grown in Dulbecco's modified essential medium (DMEM) containing $2 \mathrm{mM}$ L-glutamine, $10 \%$ fetal bovine serum, $100 \mathrm{U} / \mathrm{mL}$ penicillin, and $100 \mu \mathrm{g} / \mathrm{mL}$ streptomycin, in an incubator set at $37{ }^{\circ} \mathrm{C}$ with a humidified atmosphere containing $5 \% \mathrm{CO}_{2}$. Exponentially growing 
cancer cells were plated onto 96-well plates at 5000 cells per well in $200 \mu \mathrm{L}$ DMEM; $24 \mathrm{~h}$ later, the cells were exposed for $48 \mathrm{~h}$ to the solvent alone (1\% DMSO final concentration for the controls) or to the flavonoid at the indicated concentrations. Viability was assessed using the MTT (1-(4,5-dimethylthiazol-2yl)-3,5-diphenyltetrazolium) test, and absorbance was read at $562 \mathrm{~nm}$ in a microplate reader (BioKinetics Reader, EL340, Fisher Bioblock Scientific, Illkirch, France). Experiments were run in triplicate and repeated 3-6 times. Results are presented as the inhibitory concentrations for $50 \%\left(\mathrm{IC}_{50}\right)$ of cells for a $48 \mathrm{~h}$ exposure time.

\subsubsection{Morphologic effects on EAhy 926 endothelial cells}

To assess the effects of flavonoids on the morphology of endothelial cells, we used the EAhy 926 endothelial cell line which is derived from the fusion of human umbilical vein endothelial cells (HUVEC) with the permanent human cell line A549 [37]. The EAhy 926 cell line is considered as one of the best immortalized HUVEC cell lines because these cells express most of the biochemical markers of parental HUVEC EAhy 926 cells [38]. EAhy 926 cells were grown in DMEM containing $2 \mathrm{mM}$ L-glutamine, 10\% fetal bovine serum, $100 \mathrm{U} / \mathrm{mL}$ penicillin and $100 \mu \mathrm{g} / \mathrm{mL}$ streptomycin $\left(37{ }^{\circ} \mathrm{C}, 5 \% \mathrm{CO}_{2}\right)$. Exponentially growing EAhy 926 cells were plated onto 96 -well plates at 5000 cells $/ 100 \mu \mathrm{L} /$ well. Twenty-four hours after plating, the medium was aspirated, and $100 \mathrm{~mL}$ of medium containing the test compound was added to the well containing the cells (in triplicate) in 10-fold dilutions, and incubated for $2 \mathrm{~h}$. After the $2 \mathrm{~h}$ incubation period, digital photographs were taken of representative centre areas of each well at a magnification of 100x and 200x. The antivascular agent combretastatin A4 was routinely included in the experiments as internal standard. Results are presented as the efficacious concentration for the change in cell shape (rounding up) for more than $20 \%$ of cells after a $2 \mathrm{~h}$ exposure time $\left(\mathrm{EC}_{20}\right)$.

\section{Results and discussion}

\subsection{Synthesis of compounds}

Although ferrocenyl chalcones, where ferrocene replaces the phenyl ring in the chalcone skeleton, have been studied since the 1950s [39], only recently have ferrocene-substituted higher flavonoids been obtained. Access to these molecules was accomplished by the synthesis of the ferrocenyl aurone via an oxidative 1,5-cyclization of 2-hydroxychalcone. The latter being obtained via a condensation of ferrocenecarboxaldehyde and 2-hydroxyacetophenone, a variety of substitution patterns can be acquired by selecting appropriate commercially-available 2-hydroxyacetophenones. A series of ten ferrocene-substituted chalcones, 1a-j, with halogen and methoxy substitution were synthesized (Scheme 1) and subsequently treated with $\mathrm{Hg}(\mathrm{OAc})_{2}$ in pyridine to form the corresponding series of ferrocenyl aurones $\mathbf{2 a - \mathbf { j }}$ (cf. Table 1) [28]. Isomerization of the aurones by refluxing the latter in EtOH with KCN gave ferrocenyl flavones, 3a-j, in yields ranging from $55 \%$ to $88 \%$ (cf. Table 1) [27]. Flavonols $\mathbf{5 a}, \mathbf{5 c}$ and $\mathbf{5 g}$ were obtained from the corresponding flavones by treatment with oxone and acetone in a biphasic $\mathrm{CH}_{2} \mathrm{Cl}_{2} /$ carbonate buffer solution as previously reported [27].

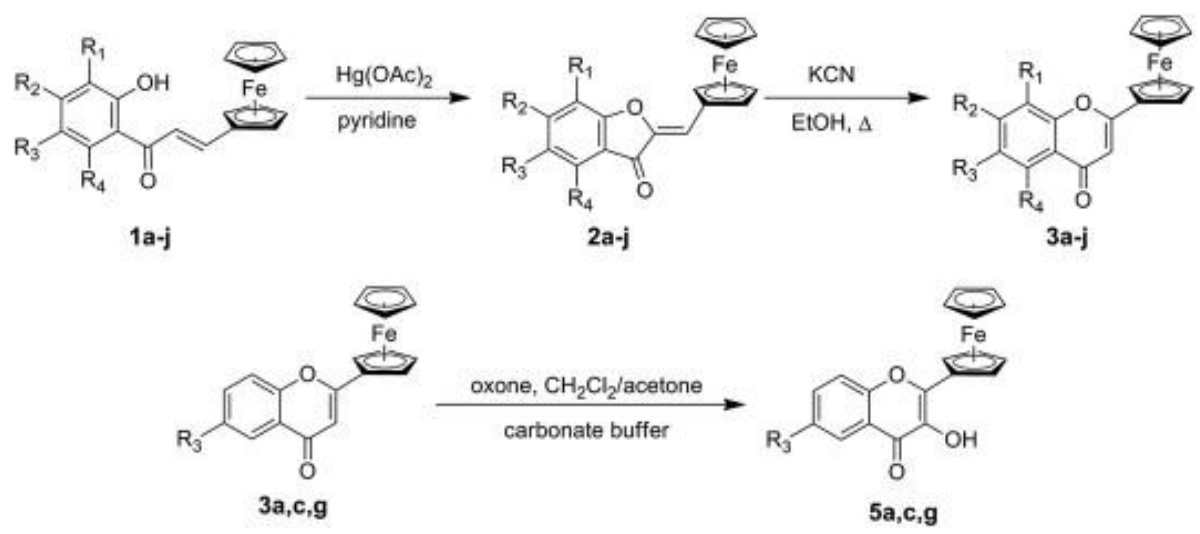


Scheme 1. Synthesis of ferrocenyl aurones, flavones and flavonols. a: $R=\mathrm{H} ; \mathrm{b}: R_{3}=\mathrm{Cl} ; \mathrm{c}: R_{3}=\mathrm{Br} ; \mathrm{d}: R_{l}=R_{3}=\mathrm{F} ; \mathrm{e}: R_{I}=R_{3}=$ $\mathrm{Cl}$; f: $R_{1}=R_{3}=\mathrm{Br} ; \mathrm{g}: R_{2}=\mathrm{OMe} ; \mathrm{h}: R_{3}=\mathrm{OMe} ; \mathrm{i}: R_{4}=\mathrm{OMe} ; \mathrm{j}: R_{2}=R_{4}=\mathrm{OMe}$.

Table 1. Cell and refinement parameters for compound $\mathbf{6 a .}$

Sum formula

Molecular weight

Crystal system

Space group

$a, \AA$

$b, \AA$

$c, \AA$

$V, \AA^{3}$

Z

$T, \mathrm{~K}$

$\lambda, \AA$

$D_{\text {calc }}, \mathrm{g} / \mathrm{cm}^{3}$

$\mu_{\mathrm{MoK} \alpha}, \mathrm{mm}^{-1}$

$F(000)$

$\theta$ range, ${ }^{\circ}$

Reflections collected

Refinement reflections/restraints/parameters

GooF on $F^{2}$

Final $R$ indices $[I>2 \sigma(I)]$

$R$ indices (all data)
$\mathrm{C}_{26} \mathrm{H}_{20} \mathrm{FeO}_{2}$

420.29

orthorhombic

Pcab

14.706(1)

$15.632(2)$

$17.146(3)$

$3941.5(8)$

8

200(2)

0.71073

1.416

0.785

1744

$2.95-30.00$

23,720

$5725 / 0 / 263$

0.9286

$R_{1}=0.0406^{\mathrm{a}}$

$w R_{2}=0.0924^{\mathrm{b}}$

$R_{1}=0.0848^{\mathrm{a}}$

$w R_{2}=0.1116^{\mathrm{b}}$

$R_{1}={ }^{a} \Sigma|| F_{0}|-| F_{C}|| / \Sigma\left|F_{0}\right|$, and $w R_{2}={ }^{\mathrm{b}}\left[\Sigma w\left(F_{0}{ }^{2}-F_{C}{ }^{2}\right)^{2} / \Sigma w\left(F_{0}^{2}\right)^{2}\right]^{1 / 2}, w=1 /\left[\sigma^{2}\left(F_{0}{ }^{2}\right)+(a P)^{2}+b P\right]$ and $P=\left(F_{0}{ }^{2}+2 F_{C}{ }^{2}\right) / 3$.

Ferrocenyl flavanones 6a-d were synthesized by coupling organic flavanones - formed by base-catalyzed cyclization of the corresponding chalcone - with ferrocene carboxaldehyde in an ethanolic solution of $\mathrm{HCl}$. The yields of these reactions were poor, due to decomposition of the desired adduct over the long reaction times necessary. Attempts to synthesize the condensation products using neat piperidine, sulfuric acid or $\mathrm{NaOH}$ in $\mathrm{EtOH}$ or diethylamine or piperidine [40] in DMF yielded no traces of the desired compounds (Scheme 2).
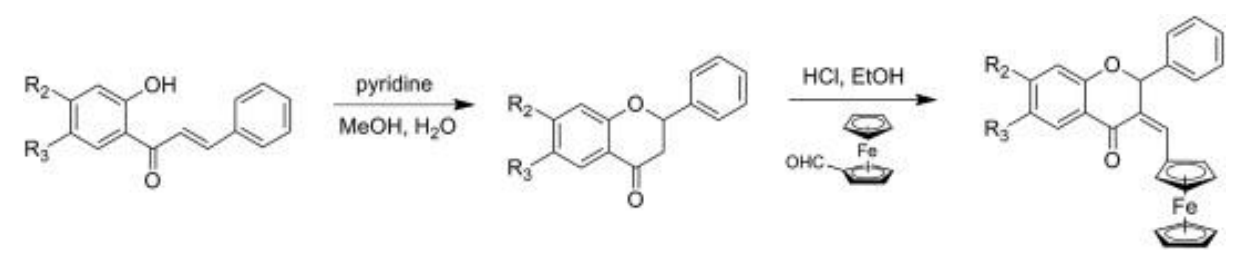

Scheme 2. Synthesis of ferrocenyl flavanones. $\mathbf{6 a}=$ unsubstituted; $\mathbf{6 b}: R_{2}=\mathrm{OMe} ; \mathbf{6 c}: R_{3}=\mathrm{OMe} ; \mathbf{6 d}: R_{3}=\mathrm{Cl}$.

One of the ferrocenyl flavanones, 6a, gave crystals suitable for X-ray analysis by slow diffusion of pentane into a saturated dichloromethane solution (Table 1). The structure of $6 \mathbf{a}$ (Fig. 1) is essentially comprised of a quasi-planar arrangement of the $\mathrm{C}_{5} \mathrm{H}_{4}$ ring of the ferrocene and the 
chromanone, while the FeCp and phenyl moieties extend below this plane. The ferrocene group is slightly twisted out of the quasi-plane of the chromanone moiety with a torsion angle of $15.5^{\circ}$ for $\mathrm{C} 2-\mathrm{C} 1-\mathrm{C} 11-\mathrm{C} 12$. All of the atoms of the chromanone share one plane, except for $\mathrm{C} 16$ and $\mathrm{O} 1$, where torsion angles of between $13.7^{\circ}$ (C14-C13-C12-C16) and 176.7 ${ }^{\circ}$ (C20-C14-C15-O1) are observed. The phenyl ring is almost perpendicular to the plane of the molecule, with an angle of $88^{\circ}$ between the plane formed by the phenyl ring and that formed by the $\mathrm{C}_{5} \mathrm{H}_{4}$-C-chromanone.

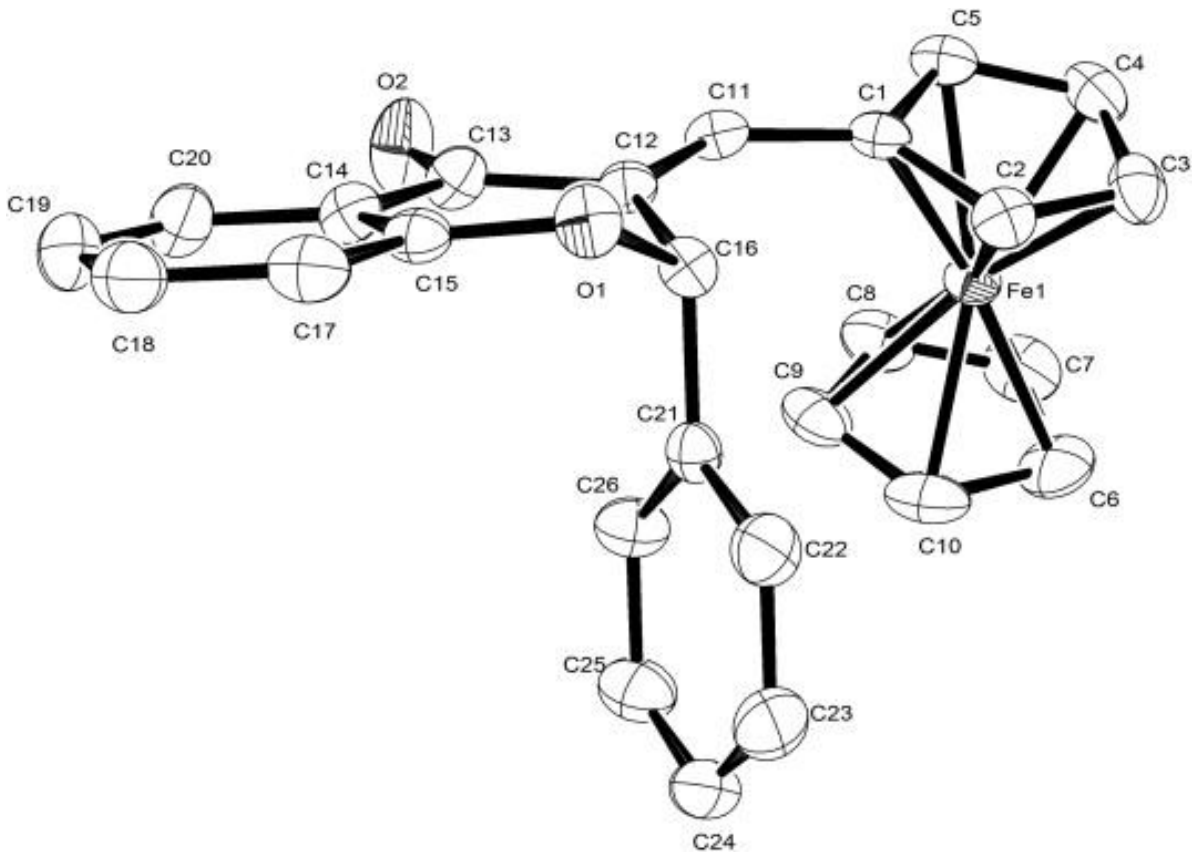

Fig. 1. Ortep diagram of 6a. Thermal ellipsoids shown at 50\% probability, hydrogen atoms omitted for clarity. Selected bond distances $(\AA): \mathrm{C} 13-\mathrm{O} 2=1.224(2) ; \mathrm{Fe} 1-\mathrm{C}_{\mathrm{Cp}}=2.027(2)-2.049(2)($ range $), 2.036(2)(\mathrm{avg}) ; \mathrm{C} 1-\mathrm{C} 11=1.439(3) ; \mathrm{C} 11-$

$\mathrm{C} 12=1.339(3)$; and angles $\left({ }^{\circ}\right): \mathrm{Cp}_{\text {centroid }}-\mathrm{Fe}-\mathrm{Cp}_{\text {centroid }}=178.10^{\circ} ; \mathrm{C} 1-\mathrm{C} 11-\mathrm{C} 12=131.05(18)^{\circ} ; \mathrm{C} 13-\mathrm{C} 12-\mathrm{C} 16-\mathrm{O} 1=-41.13^{\circ}$.

\subsection{Cytotoxicity of ferrocenyl chalcones, aurones and flavones}

The antiproliferative effects, expressed as $\mathrm{IC}_{50}$ values, of the ferrocenyl chalcones, aurones and flavones against B16 melanoma cells for a $48 \mathrm{~h}$ exposure time, are presented in Table 2. For the compounds where $\mathrm{R}=\mathrm{H}$ or $\mathrm{OMe}$, the geometry of the flavonoid and the number or position of the substituents have only a modest effect on the cytotoxicity. For example, the unsubstituted compounds 1a, 2a and 3a presented similar activity, with an average $\mathrm{IC}_{50}$ value of $44 \pm 5 \mu \mathrm{M}$. The methoxylated compounds $\mathbf{1 g}-\mathbf{j}, \mathbf{2 g}-\mathbf{j}$ and $\mathbf{3 g}-\mathbf{j}$, all have $\mathrm{IC}_{50}$ values ranging within a factor of 2 (between 36 and $63 \mu \mathrm{M}$ ), except for the bismethoxychalcone $\mathbf{1 j}$, which is not active at $100 \mu \mathrm{M}$. 
Table 2. Cytotoxicity on B16 murine melanoma cells ( $\mathrm{IC}_{50}$ in $\left.\mu \mathrm{M}\right)$ and lowest concentration causing morphological changes on EAhy 926 endothelial cells $\left(\mathrm{EC}_{20}\right.$ in $\left.\mu \mathrm{M}\right)$ of ferrocenyl compounds.

\begin{tabular}{|c|c|c|c|c|c|c|c|c|c|}
\hline & & $\mathrm{IC}_{50}(\mu \mathrm{M})^{\mathrm{a}}$ & $\mathbf{E C}_{20}(\mu \mathrm{M})^{\mathrm{b}}$ & & $\mathrm{IC}_{50}(\mu \mathrm{M})^{\mathrm{a}}$ & $\mathbf{E C}_{20}(\mu \mathrm{M})^{\mathrm{b}}$ & & $\mathrm{IC}_{50}(\mu \mathrm{M})^{\mathrm{a}}$ & $\mathbf{E C}_{20}(\mu \mathrm{M})^{\mathrm{b}}$ \\
\hline Unsubstituted & 1a & $50 \pm 1$ & $>100$ & $2 a$ & $45 \pm 4$ & 25 & $3 a$ & $39 \pm 1$ & 25 \\
\hline$R_{3}=\mathrm{Cl}$ & $1 b$ & $30 \pm 2$ & $>100$ & $2 b$ & $16 \pm 1$ & $>100$ & $3 \mathbf{b}$ & $41 \pm 1$ & 1.56 \\
\hline$R_{3}=\mathrm{Br}$ & $1 \mathrm{c}$ & $45 \pm 3$ & $>100$ & $2 c$ & $16 \pm 1$ & $>100$ & $3 c$ & $41 \pm 1$ & 0.8 \\
\hline$R_{1}=R_{3}=\mathrm{F}$ & 1d & $49 \pm 1$ & 12.5 & 2d & $18 \pm 1$ & $>100$ & 3d & $54 \pm 2$ & 100 \\
\hline$R_{1}=R_{3}=\mathrm{Cl}$ & 1e & $74 \pm 3$ & 50 & $2 e$ & $12 \pm 1$ & $>100$ & $3 e$ & $55 \pm 4$ & 6.25 \\
\hline$R_{1}=R_{3}=\mathrm{Br}$ & 1f & $84 \pm 5$ & 25 & $2 f$ & $14 \pm 1$ & 50 & 3f & $91 \pm 8$ & 0.8 \\
\hline$R_{2}=\mathrm{OMe}$ & $1 \mathrm{~g}$ & $43 \pm 2$ & 50 & $2 \mathrm{~g}$ & $48 \pm 1$ & 100 & $3 g$ & $36 \pm 1$ & 50 \\
\hline$R_{3}=\mathrm{OMe}$ & $1 \mathrm{~h}$ & $39 \pm 4$ & 50 & $2 h$ & $46 \pm 2$ & 6.25 & $3 h$ & $38 \pm 1$ & 12.5 \\
\hline$R_{4}=\mathrm{OMe}$ & $\mathbf{1 i}$ & $42 \pm 2$ & 50 & $2 \mathbf{i}$ & $53 \pm 2$ & 6.25 & $3 \mathbf{i}$ & $63 \pm 2$ & 6.25 \\
\hline$R_{2}=R_{4}=\mathrm{OMe}$ & $\mathbf{1 j}$ & $>100$ & 50 & $2 \mathbf{j}$ & $46 \pm 1$ & 50 & $\mathbf{3 j}$ & $49 \pm 2$ & $>100$ \\
\hline Mean $\mathrm{IC}_{50}$ & & $56 \pm 44$ & & & $31 \pm 22$ & & & $51 \pm 40$ & \\
\hline
\end{tabular}

The compounds comprising the halogen series have a more diverse profile. The halogenated chalcones and flavones show low activity, with $\mathrm{IC}_{50}$ values ranging between $30 \mu \mathrm{M}$ and $91 \mu \mathrm{M}$. However, the aurones $\mathbf{2 b - f}$ all show cell growth inhibition at concentrations below $20 \mu \mathrm{M}$. The $\mathrm{IC}_{50}$ values are similar, regardless of the type of halogen substitution, ranging between $12 \mu \mathrm{M}$ and $18 \mu \mathrm{M}$.

Indeed, it is the presence of the active halogenated aurones which drive down the mean $\mathrm{IC}_{50}$ value for the aurone class. The ferrocenyl chalcones and flavones, on the other hand, have a similar mean and range of activity $(55.5 \pm 44.5$ and $50.6 \pm 40.6 \mu \mathrm{M}$, respectively). We should mention that B16 cells seem to be particularly resistant to these compounds. Previous studies [28] on MDA-MB-231 breast cancer cells found $\mathrm{IC}_{50}$ values in the low micromolar range $(1-4 \mu \mathrm{M})$ for methoxylated aurones $\mathbf{2 g - j}$.

\subsection{Morphological effects of ferrocenyl chalcones, aurones and flavones}

The effect of these compounds on the morphology of endothelial cells (EAhy 926), which could be predictive of antivascular activity, was assessed. Fig. 2 shows the characteristic "rounding up" of endothelial cells after a brief 2-h exposure time to a representative active flavonoid compared to controls. It can be observed that compound $\mathbf{3 b}$ causes a rounding up of endothelial cells for $53 \%$ of cells at a concentration of 3 $\mu \mathrm{M}$, whereas $14 \%$ of control cells adopted a round shape.

Remarkably, many of the compounds showed morphological activity after a $2 \mathrm{~h}$ exposure time at non-cytotoxic concentrations. The exposure time was deliberately chosen to be short to simulate more closely the acute effects of these flavonoids upon in vivo administration. Because most of these compounds 
have a short half-life the endothelial cells are therefore exposed to high concentrations for a short time in vivo.

The ferrocenyl flavonoids show a wide range in morphological activity, and the geometry of the flavonoid skeleton has an important impact on their potency. The ferrocenyl chalcones have little effect on the endothelial cells, with only two molecules, $\mathbf{1 d}$ and $\mathbf{1 f}$, showing activity at concentrations below $50 \mu \mathrm{M}$. The ferrocenyl aurones are more effective, with three compounds changing the cell morphology at concentrations below $50 \mu \mathrm{M}$, and two, $\mathbf{2 h}$ and $\mathbf{2 i}$, showing activity at $6.25 \mu \mathrm{M}$. The flavones show by far the best morphological activity of all of the ferrocenyl flavonoid classes examined. Indeed, most of the compounds are active at concentrations below $25 \mu \mathrm{M}$. Most notably, the halogenated compounds $\mathbf{3 b}, \mathbf{3 c}$ and 3f, are active at low or submicromolar concentrations, which compare favorably to compounds in the combretastatin series, which are considered as the reference compounds in the antivascular series [41].

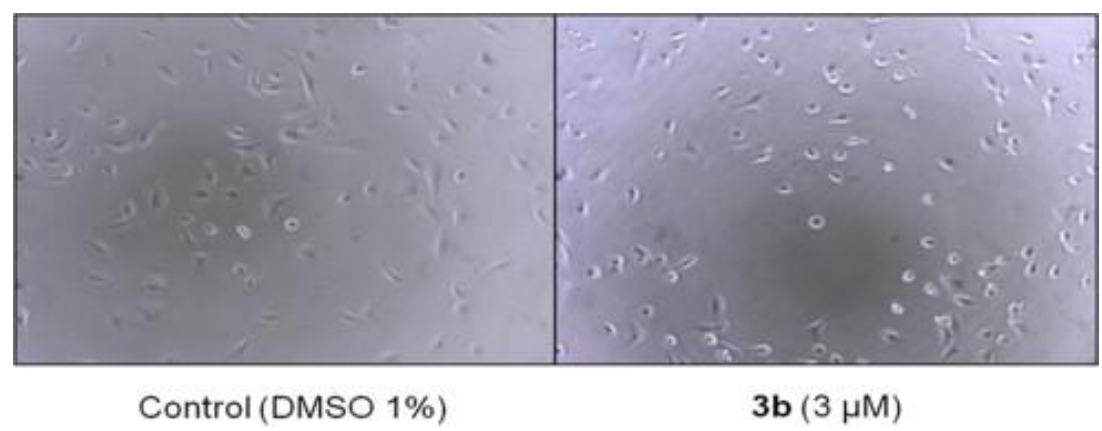

Fig. 2. Representative photographs depicting the morphological effects of compound $\mathbf{3 b}$ on endothelial cells (EAhy 926). Cells in exponential growth were exposed for $2 \mathrm{~h}$ to either 1\% DMSO (control) or compound $\mathbf{3 b}$ at $3 \mu \mathrm{M}$. Original magnification was $360 \times$.

\subsection{Comparison of the biological effects of ferrocenyl and organic flavones}

As shown above, some of the ferrocenyl flavones can induce changes in the morphology of endothelial cells at low concentrations. To investigate the influence of the ferrocenyl group on morphological effects and cytotoxicity, the corresponding series of organic flavones was synthesized by a 1,6-oxidative cyclization of chalcones with $\mathrm{I}_{2}$ in DMSO [24]. We observed that the presence of a ferrocene in lieu of a phenyl group generally enhanced the cytotoxicity on cancer cells, as presented in Table 3 . This enhanced cytotoxicity against cancer cells has already been observed by us and by others in various ferrocene functionalized biomolecules $[12,13,18 b, 42]$.

Concerning the morphological effects on endothelial cells, the comparison of ferrocenyl flavones to their organic counterparts did not allow us to draw clear structure-activity relationships. For the compound pairs $\mathbf{3 a} / \mathbf{4} \mathbf{a}, 3 \mathbf{d} / \mathbf{4} \mathbf{d}, \mathbf{3} \mathbf{g} / \mathbf{4} \mathbf{g}$ and $\mathbf{3} \mathbf{j} / \mathbf{4} \mathbf{j}$, the presence of ferrocene inhibited the morphological activity of compound compared to its phenyl analogue. For example, the simple unsubstituted flavone 4a shows surprisingly good morphological activity on endothelial cells, whereas its ferrocenyl counterpart 3a was active at a 32-fold higher concentration. On the other hand, for the pairs of compounds $3 \mathbf{c} / \mathbf{4 c}, \mathbf{3 e} / \mathbf{4 e}, \mathbf{3 f} / \mathbf{4 f}$, $\mathbf{3 h} / \mathbf{4 h}$ and $3 \mathbf{i} / \mathbf{4} \mathbf{i}$ the ferrocenyl derivative clearly showed a stronger morphological effect on the endothelial cells compared to its unsubstituted counterpart. Therefore, in summary, while the addition of ferrocene to the ferrocenyl flavones tends to enhance the compound's cytotoxicity, there does not seem to be a clear effect on their morphological activity. 
Table 3. Cytotoxicity on B16 murine melanoma cells and lowest concentration causing morphological changes on EAhy 926 endothelial cells of organic flavones $\mathbf{4} \mathbf{a}-\mathbf{j}$ compared to ferrocenyl flavones $\mathbf{3} \mathbf{a}-\mathbf{j}$.

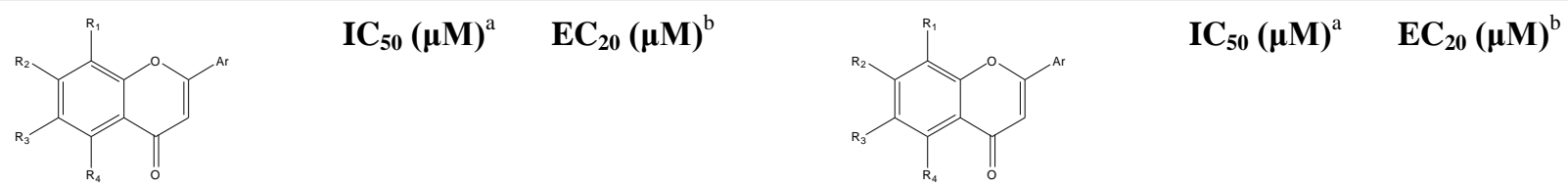

\begin{tabular}{|c|c|c|c|c|c|c|c|}
\hline \multirow[t]{2}{*}{ Unsubstituted } & $\mathbf{3 a}(\mathrm{Fc})$ & $39 \pm 1$ & 25 & $R_{1}=R_{3}=\mathrm{Br}$ & $\mathbf{3 f}(\mathrm{Fc})$ & $91 \pm 8$ & 0.8 \\
\hline & $\mathbf{4 a}(\mathrm{Ph})$ & $49 \pm 3$ & 0.78 & & $4 \mathbf{f}(\mathrm{Ph})$ & $>100$ & 6.3 \\
\hline \multirow[t]{2}{*}{$R_{3}=\mathrm{Cl}$} & $3 \mathbf{b}(\mathrm{Fc})$ & $41 \pm 1$ & 1.56 & $R_{2}=\mathrm{OMe}$ & $3 \mathbf{g}(\mathrm{Fc})$ & $36 \pm 1$ & 50 \\
\hline & 4b $(\mathrm{Ph})$ & $>100$ & n.d. & & $4 \mathbf{g}(\mathrm{Ph})$ & $>100$ & 1.5 \\
\hline \multirow[t]{2}{*}{$R_{3}=\mathrm{Br}$} & $3 \mathbf{c}(\mathrm{Fc})$ & $41 \pm 1$ & 0.8 & $R_{3}=\mathrm{OMe}$ & $3 \mathbf{h}(\mathrm{Fc})$ & $38 \pm 1$ & 12.5 \\
\hline & $4 c(P h)$ & $>100$ & 3.1 & & $\mathbf{4 h}(\mathrm{Ph})$ & $>100$ & 25 \\
\hline \multirow[t]{2}{*}{$R_{1}=R_{3}=\mathrm{F}$} & 3d $(\mathrm{Fc})$ & $54 \pm 2$ & 100 & $R_{4}=\mathrm{OMe}$ & $3 \mathbf{i}(\mathrm{Fc})$ & $63 \pm 2$ & 6.25 \\
\hline & 4d $(\mathrm{Ph})$ & $>100$ & 50 & & $\mathbf{4 i}(\mathrm{Ph})$ & $89 \pm 9$ & 12.5 \\
\hline \multirow[t]{2}{*}{$R_{1}=R_{3}=\mathrm{Cl}$} & $\mathbf{3 e}(\mathrm{Fc})$ & $55 \pm 4$ & 6.25 & $R_{2}=R_{4}=\mathrm{OMe}$ & $\mathbf{3 j}(\mathrm{Fc})$ & $49 \pm 2$ & $>100$ \\
\hline & $\mathbf{4 e}(\mathrm{Ph})$ & $>100$ & 25 & & $\mathbf{4 j}(\mathrm{Ph})$ & $98 \pm 11$ & 12.5 \\
\hline
\end{tabular}

${ }^{\mathrm{a}} \mathrm{IC}_{50}$ is the cytotoxicity for $50 \%$ of cells and was assessed using the MTT viability test for a $48 \mathrm{~h}$ exposure time.

${ }^{\mathrm{b}} \mathrm{EC}_{20}$ is the lowest concentration $(\mu \mathrm{M})$ showing more than $20 \%$ rounded cells (negative controls with $1 \%$ DMSO $\approx 15 \%$ rounded cells).

Table 4. Cytotoxicity on B16 murine melanoma cells ( $\mathrm{IC}_{50}$ in $\left.\mu \mathrm{M}\right)$ and lowest concentration causing morphological changes on EAhy 926 endothelial cells $\left(\mathrm{EC}_{20}\right.$ in $\left.\mu \mathrm{M}\right)$ of ferrocene flavonols $\mathbf{5 a}, \mathbf{5} \mathbf{b}$ and $\mathbf{5 h}$, and of ferrocenyl flavanones $(\mathbf{6} \mathbf{a}-\mathbf{c})$.

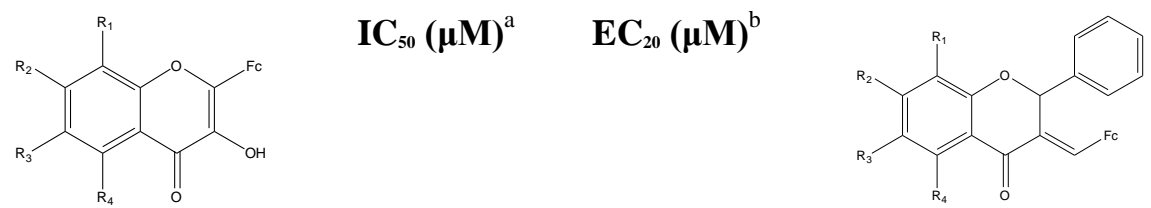

$\mathrm{IC}_{50}(\boldsymbol{\mu M})^{\mathrm{a}} \quad \mathrm{EC}_{20}(\boldsymbol{\mu M})^{\mathrm{b}}$

\begin{tabular}{llllllll}
\hline 5a & Unsub. & $>100$ & $>100$ & 6a & Unsub. & $36 \pm 2$ & 50 \\
5c & $R_{3}=\mathrm{Br}$ & $>100$ & $>100$ & 6b & $R_{2}=\mathrm{OMe}$ & $56 \pm 8$ & $>100$ \\
$\mathbf{5 h}$ & $R_{2}=\mathrm{OMe}$ & $>100$ & $>100$ & 6c & $R_{3}=\mathrm{OMe}$ & $52 \pm 7$ & 100 \\
& & & & 6d & $R_{3}=\mathrm{Cl}$ & $44 \pm 1$ & 25
\end{tabular}

${ }^{\text {a }}$ Cytotoxicity was assessed using the MTT viability test for a $48 \mathrm{~h}$ exposure time.

${ }^{\mathrm{b}}$ Lowest rounding up concentration $(\mu \mathrm{M})$ showing more than $20 \%$ rounded cells (negative controls with $1 \%$ DMSO $=15 \%$ rounded cells). 
Of the many studies undertaken on the biological properties of flavonoids, those compounds with substitution in the 3-position of the ketone, whether $\mathrm{OH}$ or a glycoside, have raised the most interest. It is therefore rather surprising that none of the three flavonols tested in this study had any effect on cell viability or cell morphology below $100 \mu \mathrm{M}$ (Table 4). This is in contradiction with the behavior of natural flavonoids. From a biosynthesis point of view, natural 3-substituted flavonoids, such as flavonols and isoflavones, possessing respectively a hydroxyl and an aromatic ring in this position, are more "evolved" than the others, since their biosynthetic enzymes appeared last [43]. Currently, the majority of natural flavonoids possess substitutions in the 3-position. Moreover, flavonols and flavanonols possess a 1,2-ketol motif, which allows chelation of certain metals, such as iron or zinc [44], known to have an important role in many biological functions.

Likewise, no antitumoral or antivascular effects were observed for the flavanones possessing a ferrocenylmethylidenyl group in the 3-position. This result was also unexpected, as several 3-benzylidenyl flavanones have recently exhibited $\mathrm{IC}_{50}$ values in the low to submicromolar range against cancer cells [45]. In this case the ferrocene does not seem to act as a good mimic of a phenyl ring. Moreover, the absence of activity of the 3-substituted ferrocenyl flavonoids (whether $\mathrm{OH}$ or $=\mathrm{C}$-Fc) suggest an entirely different mode of action than that of the organic compounds.

\section{Conclusions}

The cytotoxic and morphological effects of some three dozen ferrocenyl flavonoids have been reported. Some broad structure-activity relationships can be discerned. Overall, the halogenated aurones showed the best cytotoxic activity, although their morphological activity was modest. For the flavones, the presence of a ferrocenyl group led to a clear increase in cytotoxic activity on cancer cells, whereas for the morphological effects on endothelial cells, the presence of the ferrocenyl substituent could not be linked to a definite structure activity relationship. Surprisingly, the presence of substitution in the 3-position of ferrocenyl flavonoids yielded biologically inactive compounds.

Although the mechanism of action of these novel compounds is not presently known, they possibly act in a similar fashion as other flavonoids. For their cytotoxic action, flavonoids can act through a myriad of signaling pathways. For example, flavonoids and chalcones can regulate expression of VEGF, matrix metalloproteinases (MMPs), EGFR and inhibit NFkappaB, PI3-K/Akt, ERK1/2 signaling pathways [46]. Regarding the flavonoids morphological activity on endothelial cells, it appears that interaction with the tubulin is needed and results in a disorganization of the cytoskeleton which ultimately cause the endothelial cell to adopt a round shape. This cell morphology ultimately may translate into an antitumoral antivascular action in vivo [47].

Overall, there was no correlation observed between cytotoxic effects and potential antiangiogenic effects. For example, the most cytotoxic compounds, the halogenated ferrocenyl aurones, show only mild morphological effects, while the most active compounds, the chloro and bromo ferrocenyl flavones, are not very cytotoxic. Thus we expect that these ferrocenyl flavones will act as pure antivascular agents in vivo, that is, destroying tumor vasculature without directly interfering with cancer cell growth or division. Ideally, however, the most promising drug leads will possess both cytotoxic and antivascular properties, which do not seem to be correlated in this class of molecules. Thus future work will focus on optimizing the ferrocenyl flavones possessing both increased cytotoxic and morphologic activities compared to their organic analogues, such as $\mathbf{3 c}, \mathbf{3 e}, \mathbf{3 f}, \mathbf{3 h}$ and $\mathbf{3 i}$.

\section{Acknowledgements}

We thank L. Etienne and P. Rosa (Institute de Chimie de la Matière Condensée de Bordeaux) for ICP-AES measurements, X. Tournaire for synthetic assistance, M.N. Rager for NMR assistance, C. Fosse 
for MS measurements and P. Herson (Université Pierre et Marie Curie, Paris) for the X-ray structure. This study was supported by the CNRS, INSERM, the French Ministry of Research, and a grant from the French National Institute of Cancer (INCa, Boulogne Billancourt, France). KNT thanks the "Association pour la recherche contre le cancer" (ARC, Villejuif, France) for a postdoctoral fellowship.

\section{Appendix A. Supplementary data}

CCDC 906156 contains the supplementary crystallographic data for this paper. These data can be obtained free of charge from The Cambridge Crystallographic Data Centre via www.ccdc.cam.ac.uk/data_request/cif.

\section{Appendix B. Supplementary data}

Supplementary data related to this article can be found at http://dx.doi.org/10.1016/j.jorganchem.2012.12.031.

\section{References}

[1] Using the query "flavonoid" at clinicaltrials.gov on October 15, 2012, one finds some 60 clinical trials where flavonoids have been/are currently being studied.

[2] J. Molnár, H. Engi, J. Hohmann, P. Molnár, J. Deli, O. Wesolowska, K. Michalak, Q. Wang, Curr. Top. Med. Chem. 10 (2010) 1757-1768.

[3] (a) L. Gamet-Payrastre, S. Manenti, M.-P. Gratacap, J. Tulliez, H. Chap, B. Payrastre, Gen. Pharmacol. 32 (1999) 279-286;

(b) P.C. Ferriola, V. Cody, E. Middleton Jr., Biochem. Pharmacol. 38 (1989) 1617-1624.

[4] (a) B. Halder, S. Das Gupta, A. Gomes, FEBS J. 279 (2012) 2876-2891;

(b) A. Boumendjel, X. Ronot, J. Boutonnat, Curr. Drug Targets 10 (2009) 363-371.

[5] (a) F. Li, C. Li, H. Zhang, Z. Lu, Z. Li, Q. You, N. Lu, Q. Guo, Toxicol. Appl. Pharmacol. 261 (2012) 217-226;

(b) C.J. Weng, G.C. Yen, Cancer Metastasis Rev. 31 (2012) 323-351.

[6] B.C. Baguley, Clin. Lung Cancer 12 (2011) 81-86.

[7] B.C. Baguley, M.J. McKeage, Future Oncol. 6 (2010) 1537-1543.

[8] M.J. McKeage, B.C. Baguley, Cancer 116 (2010) 1859-1871.

[9] (a) M. Patra, G. Gasser, N. Metzler-Nolte, Dalton Trans. 41 (2012) 6350-6358;

(b) M. Navarro, W. Castro, C. Biot, Organometallics 31 (2012) 5715-5727.

[10] M. Patra, G. Gasser, ChemBioChem 13 (2012) 1232-1252.

[11] (a) G. Jaouen, N. Metzler-Nolte (Eds.), Medicinal Organometallic Chemistry, Springer-Verlag, Berlin Heidelberg, 2010;

(b) G. Gasser, N. Metzler-Nolte, Curr. Opin. Chem. Biol. 16 (2012) 84-91;

(c) C.G. Hartinger, N. Metzler-Nolte, P.J. Dyson, Organometallics 31 (2012) 5677-5685. 
[12] J. Spencer, J. Amin, M. Wang, G. Packham, S.S. Alwi, G.J. Tizzard, S.J. Coles, R.M. Paranal, J.E. Bradner, T.D. Heightman, ACS Med. Chem. Lett. 2 (2011) 358-362.

[13] C. Biot, N. Chavain, F. Dubar, B. Pradines, X. Trivelli, J. Brocard, I. Forfar, D. Dive, J. Organomet. Chem. 694 (2009) 845-854.

[14] E.A. Hillard, A. Vessières, L. Thouin, G. Jaouen, C. Amatore, Angew. Chem. Int. Ed. 45 (2006) 285290.

[15] W.H. Ang, A. Casini, G. Sava, P.J. Dyson, J. Organomet. Chem. 696 (2010) 989-998.

[16] Z. Liu, A. Habtemariam, A. Pizarro, S. Fletcher, A. Kisova, O. Vrana, L. Salassa, P. Bruijnincx, G. Clarkson, V. Brabec, P. Sadler, J. Med. Chem. 54 (2011) 3011-3026.

[17] S. Chatterjee, I. Biondi, P.J. Dyson, A. Bhattacharyya, J. Biol. Inorg. Chem. 16 (2011) 715-724.

[18] (a) N. Metzler-Nolte, M. Salmain, The bioorganometallic chemistry of ferrocene, in: P. Stepnicka (Ed.), Ferrocenes: Ligands, Materials and Biomolecules, Wiley, Chichester, 2008, pp. 499-639;

(b) E.A. Hillard, A. Vessières, G. Jaouen, Ferrocene functionalized endocrine modulators as anticancer agents, in: N. Metzler-Nolte, G. Jaouen (Eds.), Top. Organ. Chem., Medicinal Organometallic Chemistry, 32, Springer Verlag, Berlin Heidelberg, 2010, pp. 81-117.

[19] I. Damljanovic, M. Vukicevic, N. Radulovic, R. Palic, E. Ellmerer, Z. Ratkovic, M.D. Joksovic, R.D. Vukicevic, Bioorg. Med. Chem. Lett. 19 (2009) 1093-1096.

[20] F. Dubar, T.J. Egan, B. Pradines, D. Kuter, K.K. Ncokazi, D. Forge, J.F. Paul, C. Pierrot, H. Kalamou, J. Khalife, E. Buisine, C. Rogier, H. Vezin, I. Forfar, C. Slomianny, X. Trivelli, S. Kapishnikov, L. Leiserowitz, D. Dive, C. Biot, ACS Chem. Biol. 6 (2011) 275-287.

[21] J.-P. Monserrat, R.I. Al-Safi, K.N. Tiwari, L. Quentin, G.G. Chabot, A. Vessières, G. Jaouen, N. Neamati, E.A. Hillard, Bioorg. Med. Chem. Lett. 20 (2011) 6195-6197.

[22] (a) R. Schobert, S. Seibt, K. Mahal, A. Ahmad, B. Biersack, K. Effenberger-Neidnicht, S. Padhye, F.H. Sarkar, T. Mueller, J. Med. Chem. 54 (2011) 6177-6182;

(b) N.T. Huynh, M. Morille, J. Bejaud, P. Legras, A. Vessières, G. Jaouen, J.P. Benoit, C. Passirani, Pharmaceut. Res. 28 (2011) 3189-3198;

(c) N.T. Huynh, C. Passirani, E. Allard-Vannier, L. Lemaire, J. Roux, E. Garcion, A. Vessieres, J.-P. Benoit, Int. J. Pharma. 423 (2012) 55-62;

(d) M. Roger, A. Clavreul, N.T. Huynh, C. Passirani, P. Schiller, A. Vessières, C. Montero-Menei, P. Menei, Int. J. Pharma. 423 (2012) 63-68.

[23] For a recent review see: M. Grazul, E. Budzisz Coord. Chem. Rev. 253 (2009) 2588-2598.

[24] X. Wu, P. Wilairat, M.-L. Goa, Bioorg. Med. Chem. Lett. 12 (2002) 2299-2302.

[25] (a) L. Nagy, H. Mehner, A.A. Christy, E. Sletten, F.T. Edelmann, Q.M. Andersen, J. Radioanal. Nucl. Chem. 227 (1998) 89-98;

(b) C.E. Anson, C.S. Creaser, A.V. Malkov, L. Mojovic, G.R. Stephanson, J. Organomet. Chem. 668 (2003) 101-122;

(c) V. Guerchais, J.-L. Fillaut, Coord. Chem. Rev. 255 (2011) 2448-2457;

(d) J.-L. Fillaut, J. Andriès, R.D. Marwaha, P.-H. Lanoë, O. Lohio, L. Toupet, J.A.G. Williams, J. Organomet. Chem. 693 (2008) 228-234; 
(e) C.E. Anson, C.S. Creaser, J.A. Downie, O. Egyed, A.V. Malkov, L. Mojovic, G.R. Stephenson, A.T. Turner, K.E. Wilson, Bioorg. Med. Chem. Lett. 8 (1998) 3549-3554;

(f) W. Langseth, Inorg. Chim. Acta 90 (1984) 53-56.

[26] A. Kurzwernhart, W. Kandioller, C. Bartel, S. Bächler, R. Trondl, G. Mühlgassner, M.A. Jakupec, V.B. Arion, D. Marko, B.K. Keppler, C.G. Hartinger, Chem. Commun. 48 (2012) 4839-4841.

[27] K.N. Tiwari, J.-P. Monserrat, F. de Montigny, G. Jaouen, M.-N. Rager, E. Hillard, Organometallics 30 (2011) 5424-5432.

[28] K.N. Tiwari, J.-P. Monserrat, A. Hequet, C. Ganem-Elbaz, T. Cresteil, G. Jaouen, A. Vessières, E.A. Hillard, C. Jolivalt, Dalton Trans. 41 (2012) 6451-6457.

[29] N.N. Agrawal, P.A. Soni, Indian J. Chem. 44B (2005) 2601-2603.

[30] Z. Du, H. Ng, K. Zhang, H. Zeng, J. Wang, Org. Biomol. Chem. 9 (2011) 6930-6933.

[31] T. Patonay, J. Jeko, E. Riman, Syn. Commun. 32 (2002) 2403-2415.

[32] K. Dahlén, E.A.A. Wallén, M. Grøtli, K. Luthman, J. Org. Chem. 71 (2006) 6863-6871.

[33] M. Yoshida, Y. Fujino, T. Doi, Org. Lett. 13 (2011) 4526-4529.

[34] J.I. Lee, H.S. Son, H. Park, Bull. Korean Chem. Soc. 25 (2004) 1945-1947.

[35] G.A. Kraus, V. Gupta, Org. Lett. 22 (2010) 5278-5280.

[36] O.V. Singh, M. Muthukrishnan, Indian J. Chem. 44B (2005) 2575-2581.

[37] C.J. Edgell, C.C. McDonald, J.B. Graham, Proc. Natl. Acad. Sci. U S A 80 (1983) 3734-3737.

[38] D. Bouis, G.A. Hospers, C. Meijer, G. Molema, N.H. Mulder, Angiogenesis 4 (2001) 91-102.

[39] C.R. Hauser, J.K. Lindsay, J. Org. Chem. 22 (1957) 482-485.

[40] A. Arezki, E. Brulé, G. Jaouen, Organometallics 28 (2009) 1606-1609.

[41] N. Mur Blanch, G.G. Chabot, L. Quentin, D. Scherman, S. Bourg, D. Dauzonne, Eur. J. Med. Chem. 54 (2012) 22-32.

[42] A. Vessières, S. Top, P. Pigeon, E. Hillard, L. Boubeker, D. Spera, G. Jaouen, J. Med. Chem. 48 (2005) 3937-3940.

[43] B.S.J. Winkel, The biosynthesis of flavonoids, in: E. Grotewold (Ed.), The Science of Flavonoids, Springer, New York, 2006, pp. 71-95.

[44] P. Mladenka, K. Macáková, T. Filipský, L. Zatloukalová, L. Jahodár, P. Bovicelli, I.P. Silvestri, R. Hrdina, L. Saso, J. Inorg. Biochem. 105 (2011) 693-701.

[45] S. Huang, Y. Zhao, X.L. Zhao, Y. Wu, P. Wu, T. Liu, B. Yang, Y. Hu, X. Dong, Med. Chem. Res. (2012). http://dx.doi.org/10.1007/s00044-011-9959-8.

[46] J. Mojzis, L. Varinska, G. Mojzisova, I. Kostova, L. Mirossay, Pharmacol. Res. 57 (2008) 259-265.

[47] G.M. Tozer, C. Kanthou, B.C. Baguley, Nat. Rev. Cancer 5 (2005) 423-435. 


\section{Supporting information}

Ferrocenyl flavonoid-induced morphological modifications of

endothelial cells and cytotoxicity against B16 murine melanoma cells

Jean-Philippe Monserrat, Keshri Nath Tiwari, Lionel Quentin, Pascal Pigeon, Gérard Jaouen, Anne Vessières, Guy G. Chabot, Elizabeth A. Hillard

1H NMR characterization of organic flavones $4 \mathrm{~b}-\mathbf{j}$.

6-chloro-2-phenyl-4H-chromen-4-one, $4 \mathbf{4 b}$. $\delta_{\mathrm{H}}\left(300 \mathrm{MHz} ; \mathrm{CDCl}_{3}\right) 6.83\left(\mathrm{~s}, 1 \mathrm{H}\right.$, vinyl), 7.52-7.55 (m, 4H, $\left.\mathrm{C}_{6} \mathrm{H}_{5}\right), 7.64$ $\left(\mathrm{dd}, J=2.3 \mathrm{~Hz}, J=8.8 \mathrm{~Hz}, 1 \mathrm{H}, \mathrm{C}_{6} \mathrm{H}_{5}\right), 7.92\left(\mathrm{dd}, J=2.3 \mathrm{~Hz}, J=8.8 \mathrm{~Hz}, 2 \mathrm{H}, \mathrm{C}_{6} \mathrm{H}_{3}\right), 8.20\left(\mathrm{~d}, J=2.3 \mathrm{~Hz}, 1 \mathrm{H}, \mathrm{C}_{6} \mathrm{H}_{3}\right)$.

6-bromo-2-phenyl-4H-chromen-4-one, 4 c. $\delta_{\mathrm{H}}\left(300 \mathrm{MHz} ; \mathrm{CDCl}_{3}\right) 6.77\left(\mathrm{~s}, 1 \mathrm{H}\right.$, vinyl), $7.42\left(\mathrm{~d}, J=8.8 \mathrm{~Hz}, 1 \mathrm{H}, \mathrm{C}_{6} \mathrm{H}_{3}\right)$, 7.46-7.49 (m, 3H, $\left.\mathrm{C}_{6} \mathrm{H}_{5}\right), 7.72\left(\mathrm{dd}, J=2.2 \mathrm{~Hz}, J=8.8 \mathrm{~Hz}, 1 \mathrm{H}, \mathrm{C}_{6} \mathrm{H}_{3}\right), 7.83-7.87\left(\mathrm{~m}, 2 \mathrm{H}, \mathrm{C}_{6} \mathrm{H}_{5}\right), 8.30(\mathrm{~d}, J=2.2 \mathrm{~Hz}$, $\left.1 \mathrm{H}, \mathrm{C}_{6} \mathrm{H}_{3}\right)$.

6,8-difluoro-2-phenyl-4H-chromen-4-one, 4 d. $\delta_{\mathrm{H}}\left(300 \mathrm{MHz} ; \mathrm{CDCl}_{3}\right) 6.84\left(\mathrm{~s}, 1 \mathrm{H}\right.$, vinyl), 7.23-7.30 (m, $\left.2 \mathrm{H}, \mathrm{C}_{6} \mathrm{H}_{5}\right)$, 7.54-7.56 (m, $\left.1 \mathrm{H}, \mathrm{C}_{6} \mathrm{H}_{5}\right)$, 7.65-7.69 (m, $\left.1 \mathrm{H}, \mathrm{C}_{6} \mathrm{H}_{2}\right)$, 7.93-7.96 (m, $\left.2 \mathrm{H}, \mathrm{C}_{6} \mathrm{H}_{5} / \mathrm{C}_{6} \mathrm{H}_{2}\right)$.

6,8-dichloro-2-phenyl-4H-chromen-4-one, 4 e. $\delta_{\mathrm{H}}\left(300 \mathrm{MHz} ; \mathrm{CDCl}_{3}\right) 6.87\left(\mathrm{~s}, 1 \mathrm{H}\right.$, vinyl), $7.56\left(\mathrm{~m}, 3 \mathrm{H}, \mathrm{C}_{6} \mathrm{H}_{5}\right), 7.75$ $\left(\mathrm{d}, J=2.6 \mathrm{~Hz}, 1 \mathrm{H}, \mathrm{C}_{6} \mathrm{H}_{2}\right), 7.97-8.00\left(\mathrm{~m}, 2 \mathrm{H}, \mathrm{C}_{6} \mathrm{H}_{5}\right), 8.11\left(\mathrm{~d}, J=2.6 \mathrm{~Hz}, 1 \mathrm{H}, \mathrm{C}_{6} \mathrm{H}_{2}\right)$.

6,8-dibromo-2-phenyl-4H-chromen-4-one, 4 f. $\delta_{\mathrm{H}}\left(300 \mathrm{MHz} ; \mathrm{CDCl}_{3}\right) 6.87\left(\mathrm{~s}, 1 \mathrm{H}\right.$, vinyl), $7.56\left(\mathrm{~m}, 3 \mathrm{H}, \mathrm{C}_{6} \mathrm{H}_{5}\right), 8.00$ $\left(\mathrm{m}, 2 \mathrm{H}, \mathrm{C}_{6} \mathrm{H}_{5}\right), 8.03\left(\mathrm{~d}, J=2.2 \mathrm{~Hz}, 1 \mathrm{H}, \mathrm{C}_{6} \mathrm{H}_{2}\right), 8.30\left(\mathrm{~d}, J=2.2 \mathrm{~Hz}, 1 \mathrm{H}, \mathrm{C}_{6} \mathrm{H}_{2}\right)$.

7-methoxy-2-phenyl-4H-chromen-4-one, 4 g. $\delta_{\mathrm{H}}\left(300 \mathrm{MHz}, \mathrm{CDCl}_{3}\right) 3.90(\mathrm{~s}, 3 \mathrm{H}, \mathrm{OMe}), 6.74(\mathrm{~s}, 1 \mathrm{H}$, vinyl), $6.96(\mathrm{~m}$, $\left.2 \mathrm{H}, \mathrm{C}_{6} \mathrm{H}_{3}\right), 7.46-7.52\left(\mathrm{~m}, 3 \mathrm{H}, \mathrm{C}_{6} \mathrm{H}_{5}\right), 7.87\left(\mathrm{~m}, 3 \mathrm{H}, \mathrm{C}_{6} \mathrm{H}_{5}\right), 8.10\left(\mathrm{~d}, J=8.4 \mathrm{~Hz}, 1 \mathrm{H}, \mathrm{C}_{6} \mathrm{H}_{3}\right)$. 
6-methoxy-2-phenyl-4H-chromen-4-one, $4 \mathbf{h}$. $\delta_{\mathrm{H}}\left(300 \mathrm{MHz} ; \mathrm{CDCl}_{3}\right) 3.91(\mathrm{~s}, 3 \mathrm{H}, \mathrm{OMe}), 6.83(\mathrm{~s}, 1 \mathrm{H}$, vinyl), 7.29 (dd, $\left.J=2.8 \mathrm{~Hz}, J=8.8 \mathrm{~Hz}, 1 \mathrm{H}, \mathrm{C}_{6} \mathrm{H}_{3}\right), 7.50\left(\mathrm{~s}, 1 \mathrm{H}, \mathrm{C}_{6} \mathrm{H}_{3}\right), 7.54\left(\mathrm{~m}, 3 \mathrm{H}, \mathrm{C}_{6} \mathrm{H}_{5}\right), 7.60\left(\mathrm{~d}, J=2.8 \mathrm{~Hz}, 1 \mathrm{H}, \mathrm{C}_{6} \mathrm{H}_{3}\right), 7.91-7.94$ $\left(\mathrm{m}, 2 \mathrm{H}, \mathrm{C}_{6} \mathrm{H}_{5}\right)$.

5-methoxy-2-phenyl-4H-chromen-4-one, 4i. $\delta_{\mathrm{H}}\left(300 \mathrm{MHz} ; \mathrm{CDCl}_{3}\right) 4.00$ (s, 3H, OMe) 6.74 (s, 1H, vinyl), 6.82 (d, $J$ $\left.=8.2 \mathrm{~Hz}, 1 \mathrm{H}, \mathrm{C}_{6} \mathrm{H}_{3}\right), 7.14\left(\mathrm{dd}, J=0.9 \mathrm{~Hz}, J=8.2 \mathrm{~Hz}, 1 \mathrm{H}, \mathrm{C}_{6} \mathrm{H}_{3}\right), 7.51-7.60\left(\mathrm{~m}, 4 \mathrm{H}, \mathrm{C}_{6} \mathrm{H}_{5}\right), 7.90\left(\mathrm{~m}, 2 \mathrm{H}, \mathrm{C}_{6} \mathrm{H}_{5} / \mathrm{C}_{6} \mathrm{H}_{3}\right)$.

5,7-dimethoxy-2-phenyl-4H-chromen-4-one, 4j. $\delta_{\mathrm{H}}\left(300 \mathrm{MHz} ; \mathrm{CDCl}_{3}\right) 3.92$ (s, 3H, OMe), 3.96 (s, 3H, OMe), 6.39 $\left(\mathrm{d}, J=2.2 \mathrm{~Hz}, 1 \mathrm{H}, \mathrm{C}_{6} \mathrm{H}_{2}\right), 6.58\left(\mathrm{~d}, J=2.2 \mathrm{~Hz}, 1 \mathrm{H}, \mathrm{C}_{6} \mathrm{H}_{2}\right), 6.69$ (s, 1H, vinyl), 7.50 (m, 3H, $\left.\mathrm{C}_{6} \mathrm{H}_{5}\right), 7.89$ (dd, $J=2.2 \mathrm{~Hz}$, $\left.J=6.0 \mathrm{~Hz}, 2 \mathrm{H}, \mathrm{C}_{6} \mathrm{H}_{5}\right)$. 
Table SI1 Bond distances and angles for $6 \mathrm{a}$.

\begin{tabular}{|c|c|c|}
\hline Bond distances $(\AA)$ & Angles $\left(^{\circ}\right)$ & Angles $\left(^{\circ}\right)$ \\
\hline $\mathrm{Fe} 1 . \mathrm{C} 1.2 .0366(19)$ & $\mathrm{C} 1 . \mathrm{Fe} 1 . \mathrm{C} 2.41 .31(8)$ & $\mathrm{C} 2 . \mathrm{C} 3 . \mathrm{Fe} 1.69 .27(12)$ \\
\hline $\mathrm{Fe} 1 . \mathrm{C} 2.2 .032(2)$ & C1 . Fe1 . C3 . 69.08(8) & C2.C3 . C4 . 108.26(19) \\
\hline $\mathrm{Fe} 1 . \mathrm{C} 3.2 .043(2)$ & $\mathrm{C} 2 . \mathrm{Fe} 1 . \mathrm{C} 3.40 .69(8)$ & $\mathrm{Fe} 1 . \mathrm{C} 3 . \mathrm{C} 4.69 .58(12)$ \\
\hline $\mathrm{Fe} 1 . \mathrm{C} 4.2 .040(2)$ & $\mathrm{C} 1 . \mathrm{Fe} 1 . \mathrm{C} 4.69 .15(8)$ & $\mathrm{C} 3 . \mathrm{C} 4 . \mathrm{Fe} 1.69 .76(12)$ \\
\hline $\mathrm{Fe} 1 . \mathrm{C} 5.2 .027(2)$ & C2 . Fe1 . C4 . 68.68(9) & C3. C4 . C5 . 108.19(18) \\
\hline $\mathrm{Fe} 1 . \mathrm{C} 6.2 .032(2)$ & C3 . Fe1 . C4 . 40.66(9) & $\mathrm{Fe} 1 . \mathrm{C} 4 . \mathrm{C} 5.69 .28(12)$ \\
\hline $\mathrm{Fe} 1 . \mathrm{C} 7.2 .033(2)$ & C1. Fe1. C5 . 41.49(8) & C1.C5 . C4 . 108.81(18) \\
\hline $\mathrm{Fe} 1 . \mathrm{C} 8.2 .049(2)$ & $\mathrm{C} 2 . \mathrm{Fe} 1 . \mathrm{C} 5.69 .06(8)$ & C1. C5 . Fe1. 69.61(11) \\
\hline $\mathrm{Fe} 1 . \mathrm{C} 9.2 .032(2)$ & C3 . Fe1 . C5 . 68.40(9) & C4. C5 . Fe1 . 70.28(12) \\
\hline $\mathrm{Fe} 1 . \mathrm{C} 10.2 .031(2)$ & C4 . Fe1 . C5 . 40.44(9) & Fe1 . C6 . C7 . 69.61(14) \\
\hline $\mathrm{C} 1 . \mathrm{C} 2.1 .435(3)$ & C1 . Fe1 . C6 . 157.66(9) & Fe1 . C6 . C10 . 69.64(13) \\
\hline C1.C5 . 1.439(3) & C2 . Fe1 . C6 . 120.77(9) & C7. C6. C10.108.1(2) \\
\hline C1.C11. 1.439(3) & C3 . Fe1 . C6 . 105.75(10) & C6. C7 . Fe1 . 69.56(13) \\
\hline C2.C3 . 1.417(3) & C4 . Fe1 . C6 . 121.90(10) & C6.C7 . C8 . 108.0(2) \\
\hline C3 . C4 . 1.418(3) & C5 . Fe1 . C6 . 158.61(10) & $\mathrm{Fe} 1 . \mathrm{C} 7 . \mathrm{C} 8 \cdot 70.45(13)$ \\
\hline C4.C5 . 1.406(3) & C1 . Fe1 . C7 . 160.46(10) & C7 . C8 . Fe1 . 69.23(13) \\
\hline C6 . C7 . 1.418(4) & C2 . Fe1 . C7 . 156.64(10) & C7. C8 . C9 . 107.7(2) \\
\hline C6. C10. $1.410(4)$ & C3 . Fe1 . C7 . 120.97(10) & $\mathrm{Fe} 1 . \mathrm{C} 8$. C9 . 69.05(13) \\
\hline C7 . C8 . 1.407(4) & C4 . Fe1 . C7 . 106.67(9) & C8.C9. Fe1 . 70.34(13) \\
\hline C8. C9 . 1.416(3) & C5 . Fe1 . C7 . 123.15(9) & C8.C9 . C10 . 108.4(2) \\
\hline C9. C10.1.410(4) & C1 . Fe1 . C8 . 124.77(9) & $\mathrm{Fe} 1 . \mathrm{C} 9 . \mathrm{C} 10.69 .65(13)$ \\
\hline C11.C12. 1.339(3) & C2 . Fe1 . C8 . 161.18(10) & C6. C10.C9 . 107.7(2) \\
\hline C12.C13. 1.476(3) & C3 . Fe1 . C8 . 157.45(9) & C6. C10. Fe1 . 69.76(13) \\
\hline C12.C16. 1.495(3) & $\mathrm{C} 4 . \mathrm{Fe} 1 . \mathrm{C} 8.122 .73(9)$ & C9. C10.Fe1 . 69.74(13) \\
\hline C13.C14. 1.474(3) & C5 . Fe1 . C8 . 108.93(9) & C1. C11. C12. 131.05(18) \\
\hline $\mathrm{C} 13 . \mathrm{O} 2.1 .224(2)$ & C1 . Fe1 . C9 . 108.62(9) & C11.C12.C13. 118.21(17) \\
\hline C14.C15. 1.388(3) & C2 . Fe1 . C9 . 124.01(9) & C11.C12.C16. 124.98(18) \\
\hline C14.C20.1.397(3) & C3 . Fe1 . C9 . 159.26(10) & C13.C12.C16. 116.71(16) \\
\hline C15.C17. 1.387(3) & C4 . Fe1 . C9 . 159.43(10) & C12.C13.C14. 115.59(17) \\
\hline C15.O1. 1.360(2) & C5 . Fe1 . C9 . 124.40(9) & $\mathrm{C} 12 . \mathrm{C} 13 . \mathrm{O} 2.123 .04(18)$ \\
\hline C16.C21. 1.519(3) & $\mathrm{C} 1 . \mathrm{Fe} 1 . \mathrm{C} 10.122 .45(9)$ & C14.C13. O2 . 121.38(19) \\
\hline C16.O1 . 1.458(2) & C2 . Fe1 . C10. 106.72(9) & C13.C14.C15. 120.37(18) \\
\hline C17.C18. 1.371(3) & C3 . Fe1 . C10. 121.99(10) & C13. C14 . C20 . 120.89(19) \\
\hline C18.C19. 1.380(3) & $\mathrm{C} 4 . \mathrm{Fe} 1 . \mathrm{C} 10.158 .05(10)$ & C15.C14.C20. 118.71(18) \\
\hline C19.C20.1.372(3) & C5 . Fe1 . C10. 159.91(10) & C14.C15 . C17 . 120.46(19) \\
\hline $\mathrm{C} 21 . \mathrm{C} 22.1 .382(3)$ & C6 . Fe1 . C7 . 40.83(10) & C14.C15. O1 . 122.08(17) \\
\hline $\mathrm{C} 21 . \mathrm{C} 26.1 .382(3)$ & C6 . Fe1 . C8 . 68.14(10) & C17.C15. O1 . 117.40(18) \\
\hline $\mathrm{C} 22 . \mathrm{C} 23.1 .384(3)$ & C7 . Fe1 . C8 . 40.32(10) & C12.C16.C21. 114.47(15) \\
\hline $\mathrm{C} 23 . \mathrm{C} 24.1 .377(3)$ & C6 . Fe1 . C9 . 68.13(10) & C12.C16. O1 . 111.75(15) \\
\hline $\mathrm{C} 24 . \mathrm{C} 25.1 .367(3)$ & C7 . Fe1 . C9 . 68.21(10) & C21. C16. O1 . 108.29(14) \\
\hline \multirow[t]{14}{*}{$\mathrm{C} 25 . \mathrm{C} 26.1 .386(3)$} & C8 . Fe1 . C9 . 40.61(9) & C15 . C17 . C18 . 119.6(2) \\
\hline & C6 . Fe1 . C10 . 40.60(11) & C17.C18. C19. 120.8(2) \\
\hline & C7 . Fe1 . C10 . 68.58(10) & C18.C19.C20. 119.7(2) \\
\hline & $\mathrm{C} 8 . \mathrm{Fe} 1 . \mathrm{C} 10.68 .38(10)$ & C14.C20.C19. 120.7(2) \\
\hline & C9. Fe1 . C10 . 40.60(11) & C16.C21.C22. 119.14(18) \\
\hline & $\mathrm{Fe} 1 . \mathrm{C} 1 . \mathrm{C} 2.69 .19(11)$ & C16.C21.C26. 122.38(17) \\
\hline & $\mathrm{Fe} 1 . \mathrm{C} 1 . \mathrm{C} 5.68 .90(11)$ & C22. C21. C26. 118.47(19) \\
\hline & C2.C1 . C5 . 106.36(17) & C21.C22.C23. 120.5(2) \\
\hline & $\mathrm{Fe} 1 . \mathrm{C} 1 . \mathrm{C} 11.121 .28(14)$ & $\mathrm{C} 22 . \mathrm{C} 23 . \mathrm{C} 24.120 .2(2)$ \\
\hline & C2 . C1 . C11 . 131.32(17) & $\mathrm{C} 23 . \mathrm{C} 24 . \mathrm{C} 25.119 .9(2)$ \\
\hline & C5 . C1 . C11. 121.96(18) & $\mathrm{C} 24 . \mathrm{C} 25 . \mathrm{C} 26.119 .9(2)$ \\
\hline & $\mathrm{C} 1 . \mathrm{C} 2 . \mathrm{Fe} 1.69 .50(11)$ & C25.C26.C21. 121.0(2) \\
\hline & C1.C2.C3 . 108.38(18) & C16. O1 . C15 . 115.28(14) \\
\hline & $\mathrm{Fe} 1 . \mathrm{C} 2 . \mathrm{C} 3.70 .04(12)$ & \\
\hline
\end{tabular}

